\title{
Bridge number and Conway products
}

\author{
RYAN C BLAIR
}

\begin{abstract}
In this paper, we give a structure theorem for c-incompressible Conway spheres in link complements in terms of the standard height function on $S^{3}$. We go on to define the generalized Conway product $K_{1} *_{c} K_{2}$ of two links $K_{1}$ and $K_{2}$. Provided $K_{1} *_{c} K_{2}$ satisfies minor additional hypotheses, we prove the lower bound $\beta\left(K_{1} *_{c} K_{2}\right) \geq \beta\left(K_{1}\right)-1$ for the bridge number of the generalized Conway product where $K_{1}$ is the distinguished factor. Finally, we present examples illustrating that this lower bound is tight.
\end{abstract}

57M25, 57M27, 57M50

\section{Introduction}

Bridge number is the fewest number of maxima in any projection of a link $K$. This classical invariant is denoted $\beta(K)$ and was introduced by Schubert in his paper [5] Über eine Numerische Knoteninvariante. Schubert proves that, given a composite knot $K$ with summands $K_{1}$ and $K_{2}$, the bridge number of $K$ satisfies the following equation:

$$
\beta(K)=\beta\left(K_{1}\right)+\beta\left(K_{2}\right)-1 .
$$

The techniques used in this paper are inspired by Schultens' more modern and more elegant proof of the same equality [6].

The classical Conway sum and Conway product were originally defined in [1] as operations which received as input two tangle diagrams and produced as output a new tangle diagram. These original operations have inspired several related constructions. In [2], Lickorish studies a method of producing prime links by identifying together the boundaries of prime tangles. Scharlemann and Tomova's operation takes two links, evacuates untangles from the links' complements to form two tangles, and identifies together the boundaries of these two tangles to form a new link [4]. The definition of generalized Conway product used in this paper encapsulates the construction in [4]. By carefully choosing the two untangles and the gluing map, Scharlemann and Tomova showed the existence of a generalized Conway product which respects bridge surfaces. They go on to prove that the following inequality holds for such a product:

$$
\beta\left(K_{1} *_{c} K_{2}\right) \leq \beta\left(K_{1}\right)+\beta\left(K_{2}\right)-1
$$


However, it is also shown in [4] (via a construction by the author) that the above inequality is not always an equality, so a lower bound is needed.

The main goal of this paper is to present a lower bound on the bridge number of the generalized Conway product in terms of the bridge number of the factor links.

As an intermediary step to achieving this goal, we prove a structure theorem for cincompressible Conway spheres. A sphere $C$ embedded in $S^{3}$ which meets a link $K$ transversely in four points is called a Conway sphere. Loosely speaking, $C$ is worm-like if it is the boundary of a regular neighborhood of an arc in $S^{3}$ and meets $K$ in exactly two points near each of the endpoints of this arc. A rigorous definition of worm-like will ultimately rely on how $C$ is embedded with respect to $h$, the standard height function on $S^{3}$. The definition of worm-like and a proof of the following structure theorem are presented in Section 5.

Theorem A If $C$ is a c-incompressible Conway sphere embedded in the complement of a link $K$ in $S^{3}$ and bridge position for $K$ is thin position, then there is an isotopy of $C$ and $K$ resulting in $\left.h\right|_{K}$ having $\beta(K)$ maxima and $C$ being worm-like.

The main theorem appears below and is proven in Section 6. Also, in this section, we give the definition of generalized Conway product and distinguished factor.

Theorem B If $K_{1} *_{c} K_{2}$ is a generalized Conway product such that $C$ is c-incompressible and bridge position for $K_{1} *_{c} K_{2}$ is thin position, then $\beta\left(K_{1} *_{c} K_{2}\right) \geq \beta\left(K_{1}\right)-1$ where $K_{1}$ is the distinguished factor.

In Section 7, we provide examples that show this lower bound is tight.

I am grateful to Martin Scharlemann for suggesting that I investigate the relationship between Conway products and bridge number and for many helpful conversations.

\section{Definitions and preliminaries}

In this paper, $K$ will denote a tame, nonsplit link embedded in $S^{3}$ and $h: S^{3} \rightarrow$ $\mathbb{R} \cup\{-\infty,+\infty\}$ is a height function with level sets consisting of $2-$ spheres and two exceptional points corresponding to $+\infty$ and $-\infty$. We require that $h$ restricts to a Morse function on $K$.

Definition 2.1 If the maxima of $\left.h\right|_{K}$ occur above all of the minima, then $K$ is in bridge position. The fewest number of maxima of $\left.h\right|_{K}$ over all embeddings of $K$ is the bridge number of $K$, denoted $\beta(K)$. 
We will use the structure afforded us by the height function $h$ to study a Conway sphere $C$ in the complement of a link $K$ in the following way. Let $F_{C}$ be the singular foliation on the Conway sphere $C$ induced by $\left.h\right|_{C}$. A saddle is any leaf of this foliation homeomorphic to the wedge of two circles. By standard position, we can assume that all saddles of $F_{C}$ are disjoint from $K$.

A detailed analysis of the embedding of $C$ near a given saddle will be integral to the argumentation that follows. As such, we fix the following notation.

Any given saddle $\sigma=s_{1}^{\sigma} \vee s_{2}^{\sigma}$, lies in a level sphere $S_{\sigma}=h^{-1}(h(\sigma))$. Let $D_{1}^{\sigma}$ be the closure of the component of $S_{\sigma}-s_{1}^{\sigma}$ that is disjoint from $s_{2}^{\sigma}$ and $D_{2}^{\sigma}$ be the closure of the component of $S_{\sigma}-s_{2}^{\sigma}$ that is disjoint from $s_{1}^{\sigma}$.

A subdisk $D$ in $F_{C}$ is monotone if its boundary is entirely contained in a leaf of $F_{C}$ and the interior of $D$ is disjoint from every saddle in $F_{C}$. In practice, we will use the term subdisk in a slightly broader sense, allowing $\partial(D)$ to be immersed in $C$, where if $\partial(D)$ is immersed, then $\partial(D)$ denotes the saddle. We say a monotone disk is outermost if its boundary is $s_{i}^{\sigma}$ for some saddle $\sigma$ and label the disk $D_{\sigma}$. Similarly, if $s_{i}^{\sigma}$ bounds an outermost disk $D_{\sigma}$, we say $\sigma$ is an outermost saddle. It is usually the case that only one of $s_{1}^{\sigma}$ and $s_{2}^{\sigma}$ is the boundary of an outermost disk, so, our convention is to relabel so that $\partial\left(D_{\sigma}\right)=s_{1}^{\sigma}$.

Suppose $\sigma$ is an outermost saddle. $S_{\sigma}$ cuts $S^{3}$ into two 3 -balls. The one that contains $D_{\sigma}$ is again cut by $D_{\sigma}$ into two 3-balls $B_{\sigma}$ and $B_{\sigma}^{\prime}$. We chose the labeling of $B_{\sigma}$ and $B_{\sigma}^{\prime}$ so that $\partial\left(B_{\sigma}\right)=D_{1}^{\sigma} \cup D_{\sigma}$.

We say $\sigma$ is an inessential saddle if $\sigma$ is an outermost saddle and $D_{\sigma}$ is disjoint from $K$. An $n$-punctured disk denotes a disk embedded in $S^{3}$ that meets $K$ transversely in exactly $n$ points. An embedded simple closed curve in a Conway sphere $C$ is $c$ inessential if it bounds a 1 -punctured disk in $C$. Similarly, $\sigma$ is a $c$-inessential saddle if $\sigma$ is an outermost saddle and $D_{\sigma}$ meets $K$ exactly once.

We say a saddle $\sigma$ in $F_{C}$ is standard if there is a monotone disk $E_{\sigma}$ such that $\partial\left(E_{\sigma}\right)=\sigma$. If $\sigma$ is a standard saddle, $A_{\sigma}$ is the 3-ball with boundary $E_{\sigma} \cup D_{1}^{\sigma} \cup D_{2}^{\sigma}$ that lies completely to one side of $S_{\sigma}$.

By general position arguments, we can assume every saddle $\sigma$ in $F_{C}$ has a bicollared neighborhood in $C$ that is disjoint from $K$ and all other singular leafs of $F_{C}$. The boundary of this bicollared neighborhood consists of three circles $c_{1}^{\sigma}, c_{2}^{\sigma}$, and $c_{3}^{\sigma}$ where $c_{1}^{\sigma}$ and $c_{2}^{\sigma}$ are parallel to $s_{1}^{\sigma}$ and $s_{2}^{\sigma}$ respectively. We can assume $c_{1}^{\sigma}, c_{2}^{\sigma}$, and $c_{3}^{\sigma}$ are level with respect to $h$ and that $c_{1}^{\sigma}$ and $c_{2}^{\sigma}$ lie in the same level surface.

When there is no confusion as to which saddle we are referring, we will drop the superscripts and subscripts of $\sigma$. Figure 1 illustrates all of the terminology outlined above. 


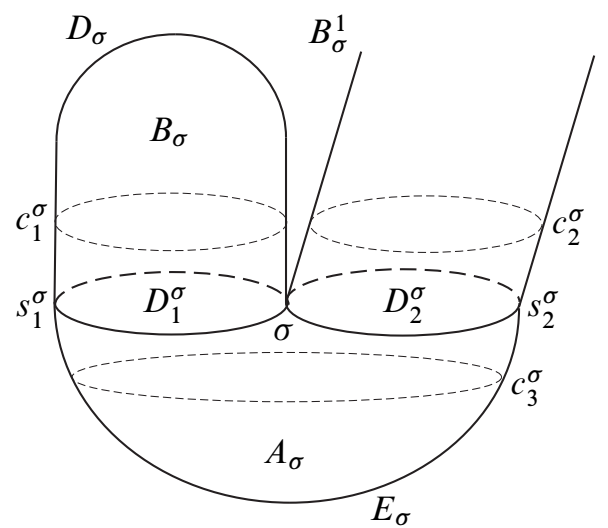

Figure 1

\section{Conway spheres}

Definition 3.1 Following [6], say a Conway sphere, $C$, is taut if the number of saddles in $F_{C}$ is minimal subject to the condition that $\left.h\right|_{K}$ has $\beta(K)$ maxima.

The goal of this section is to demonstrate the connection between the tautness of $C$ and the existence of inessential and c-inessential saddles in $F_{C}$. Most of the results will be devoted to generalizing Schultens work on companion tori in link complements [6] to the case of Conway spheres. As we will see, the condition that $C$ be taut precludes inessential saddles and some c-inessential saddles. In the following sections, we will assume additional hypotheses on the nature of bridge position of $K$ and on the Conway sphere $C$. Under these additional hypotheses we will show that a taut Conway sphere has no inessential and no c-inessential saddles.

Lemma 3.2 and its proof are immediate generalizations of Schultens' Lemma 1 in [6]. We need alter the statement and proof only slightly to account for punctures in the Conway sphere.

Lemma 3.2 Let $\sigma$ be an outermost saddle in $F_{C}$. After an isotopy of $C$ that does not change the number of saddles in $F_{C}$ and leaves both $\sigma$ and $K$ fixed, $B_{\sigma}$ does not contain $+\infty$ or $-\infty$.

Proof Without loss of generality we will assume $D_{\sigma}$ has a unique maximum $a$ (by general position, we can take $a$ to be distinct from any points in $K \cap C$ ). If $B_{\sigma}$ does not contain $+\infty$, then we are done. 
Suppose $B_{\sigma}$ contains $+\infty$ and $\alpha$ is a monotone arc with endpoints $a$ and $+\infty$ that misses $K$ and intersects $C$ only at local maxima. Label the points of $\alpha \cap C$ in order of increasing height with $a, a_{1}, \ldots, a_{n}$. See Figure 2. Again by general position, we can assume all of the $a_{i}^{\prime} s$ do not lie on $K$. Let $S_{+}$be a level sphere contained in a small neighborhood of $+\infty$ such that $S_{+}$does not meet $C$ or $K$. Let $\beta_{n}$ be a subarc of $\alpha$ with endpoints $a_{n}$ and $+\infty$. Enlarge $\beta_{n}$ slightly to be a vertical solid cylinder $V$ such that $\partial(V)$ consists of a small neighborhood of $a_{n}$ in $C$, a small disk in $S_{+}$and an annulus $A$ with $F_{A}$ being a collection of circles. Replacing $C$ with the Conway sphere $(C-V) \cup A \cup\left(S_{+}-V\right)$ represents an isotopy of $C$ in $S^{3}-K$ that does not change the number of saddles in $F_{C}$.
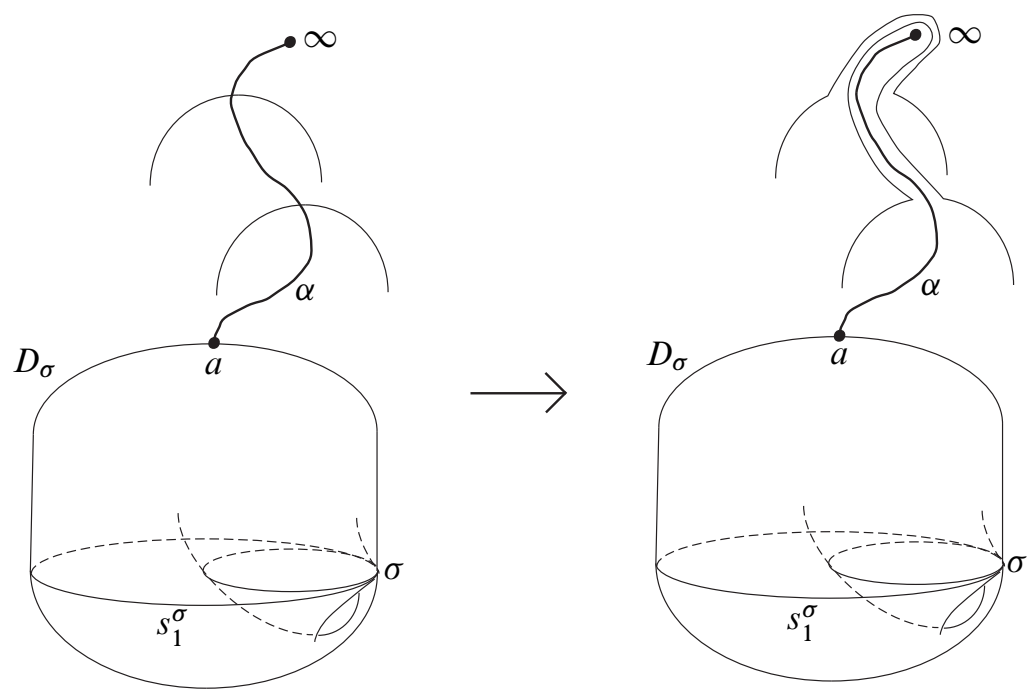

Figure 2

By induction on $n$, we can assume $\alpha$ is disjoint from $C$ except at the point $a$. By isotoping $D_{\sigma}$ to a new disk $D_{\sigma}^{*}$ in the manner described above, we have enlarged $B_{\sigma}^{\prime}$ to contain $+\infty$ and shrunk $B_{\sigma}$ so that it is disjoint from $+\infty$. After a small tilt so that $h$ again restricts to a Morse function on $D_{\sigma}^{*}, F_{D_{\sigma}^{*}}$ is a collection of circles and one maximum. The resulting Conway sphere $C^{*}$ is isotopic to $C$ via an isotopy that leaves $\sigma$ and $K$ fixed and does not change the number of saddles of $F_{C}$.

Lemma 3.3 If $F_{C}$ contains an inessential saddle, then $C$ is not taut.

Proof outline (This is Schultens' Lemma 2 in [6].) Suppose $F_{C}$ contains an inessential saddle $\sigma$. Use Lemma 3.2 to ensure $B_{\sigma}$ does not contain $+\infty$ or $-\infty$. Isotope $B_{\sigma} \cap C$ 
and $B_{\sigma} \cap K$ out of $B_{\sigma}$ in a level preserving way. Now that $B_{\sigma}$ is disjoint from $C$ and $K$, isotope $D_{\sigma}$ to $D_{1}^{\sigma}$ via $B_{\sigma}$. After a small tilt, we have eliminated $\sigma$ without introducing new critical points to $\left.h\right|_{K}$ or new saddles to $F_{C}$. We conclude $C$ is not taut.

Definition 3.4 We say $\sigma$ is a removable saddle if $\sigma$ is an outermost saddle where $D_{\sigma}$ has a unique maximum (minimum) and $\left.h\right|_{K \cap B_{\sigma}}$ has a local end-point maximum (minimum) at every point of $K \cap D_{\sigma}$. See Figure 3. Otherwise, we say $\sigma$ is nonremovable.

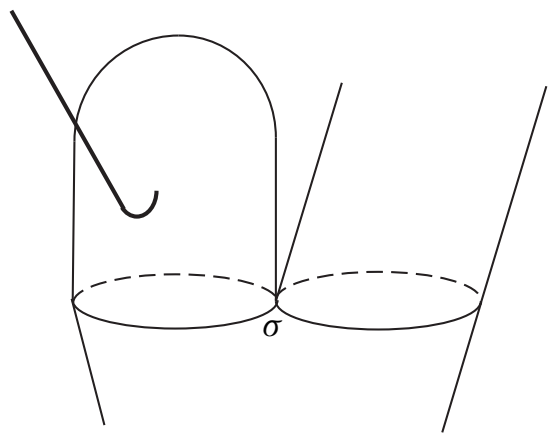

Figure 3

Lemma 3.5 If $F_{C}$ contains a removable saddle, then $C$ is not taut.

Proof Assume $\sigma$ is a removable saddle in $F_{C}$. Without loss of generality, we can assume $D_{\sigma}$ has a unique maximum and $\left.h\right|_{K \cap B_{\sigma}}$ has a local maxima at each of $\left\{k_{1}, k_{2}, \ldots, k_{n}\right\}=D_{\sigma} \cap K$. By appealing to the isotopy in Lemma 3.2, we can assume that $B_{\sigma}$ does not contain $+\infty$. Since $D_{\sigma}$ is a monotone disk, $(K \cup C) \cap \operatorname{int}\left(B_{\sigma}\right)$ can be shrunk horizontally and subsequently lowered to lie just below $D_{1}$. This isotopy does not change the number of saddles of $F_{C}$ nor the number of maxima of $\left.h\right|_{K}$, however, it does produce a collection of monotone arcs connecting every $k_{i}$ to the image of $K \cap \operatorname{int}\left(B_{\sigma}\right)$ under the isotopy. See Figure 4.

The union $D_{1} \cup D_{\sigma}$ now bounds a 3-ball minus a collection of monotone arcs, each of which has one endpoint on $D_{1}$ and one endpoint on $D_{\sigma}$. Isotope $D_{\sigma}$ to $D_{1}$ while fixing $K$ to create $C^{*}$. After a small tilt, we have produced a new Conway sphere $C^{*}$ which is isotopic to $C$ while preserving the number of maxima of $\left.h\right|_{K}$. Since the number of saddles of $F_{C^{*}}$ is one less than the number of saddles of $F_{C}, C$ is not taut. 

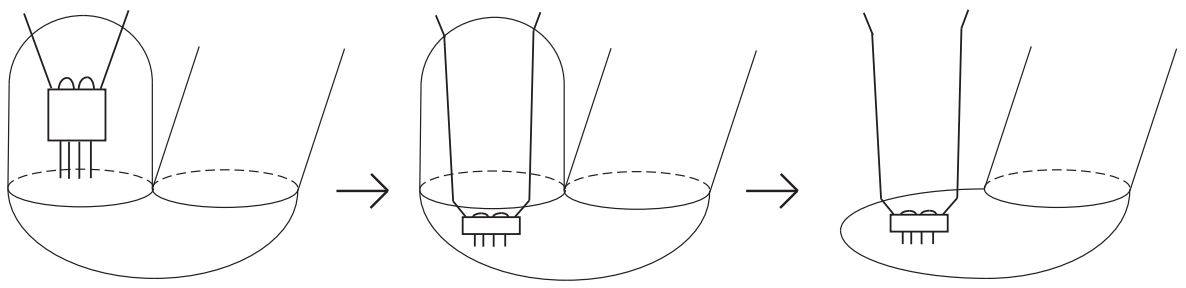

Figure 4

Since $\left.h\right|_{K}$ has $\beta(K)$ maxima, we can decompose $K$ into $2 \beta(K)$ monotone arcs. Hence, $K=\bigcup_{i=1}^{2 \beta(K)} \gamma_{i}$ where $\gamma_{i}$ has one endpoint a maximum and one endpoint a minimum of $\left.h\right|_{K}$. If $\sigma$ is a c-inessential saddle in $F_{C}$, then let $\gamma_{\sigma}$ be the $\gamma_{i}$ which meets $D_{\sigma}$.

Lemma 3.6 If $F_{C}$ contains a nonremovable $c$-inessential saddle $\sigma$ such that $D_{\sigma}$ has a maximum (minimum) and $\gamma_{\sigma}$ is disjoint from the Conway sphere $C$ above (below) $K \cap D_{\sigma}$, then $C$ is not taut.

Proof Without loss of generality we can assume $D_{\sigma}$ has a unique maximum and $\left.h\right|_{K \cap B_{\sigma}}$ has a local minimum at $p_{\sigma}=K \cap D_{\sigma}$. Let $\partial\left(\gamma_{\sigma}\right)=\left\{x_{1}, x_{2}\right\}$ where $x_{1}$ is the highest point on $\gamma_{\sigma}$ and $x_{2}$ is the lowest. Let $\alpha$ be the subarc of $\gamma_{\sigma}$ connecting $p_{\sigma}$ to $x_{1}$. Since $\gamma_{\sigma}$ is disjoint from $C$ above $K \cap D_{\sigma}$, we can choose $\mu(\alpha)$ to be a small neighborhood of $\alpha$ such that $\mu(\alpha)$ is disjoint from $C$ except in a small neighborhood of $p_{\sigma}$ on $C$ and disjoint from $K$ except for a small neighborhood of $\alpha$ in $K$. $C$ cuts $\partial(\mu(\alpha))$ into two 1-punctured disks $E$ and $F$ where $E$ is mostly above $p_{\sigma}$ and $F$ is mostly below. The 1-punctured disks $\mu(\alpha) \cap C$ and $E$ cobound a 3-ball minus a monotone arc. Hence, there is an isotopy taking $\mu(\alpha) \cap C$ to $E$ that fixes $K$ and results in a new Conway sphere $C^{*}$ which is isotopic to $C$ but has exactly one new inessential saddle $\varsigma$ such that $s_{1}^{\varsigma}$ lies in $D_{\sigma}$ just above $p_{\sigma}$ and $s_{2}^{\varsigma}$ lies in $E$. $\varsigma$ is inessential since $s_{1}^{\varsigma}$ bounds a outermost disk $D_{\varsigma}$ (the portion of $D_{\sigma}$ above $p_{\sigma}$ ). See Figure 5. It is also important to note that, after this isotopy, $\left.h\right|_{K \cap B_{\sigma}}$ now has a local maximum at $K \cap D_{\sigma}$, though, $D_{\sigma}$ is no longer a monotone disk. Using the isotopies in Lemma 3.2 and Lemma 3.3, we can produce another Conway sphere $C^{* *}$ by isotoping the disk $D_{\varsigma}$ to be level whereby we eliminate $\varsigma$ without altering the number or nature of maxima of $\left.h\right|_{K}$.

Since $C^{*}$ had one more saddle than $C$ and $C^{* *}$ has one less saddle than $C^{*}$, then $C$ and $C^{* *}$ have the same number of saddles. The aggregate isotopy from $C$ to $C^{* *}$ fixes $\sigma$, preserves the number of saddles of the Conway sphere, preserves the number 


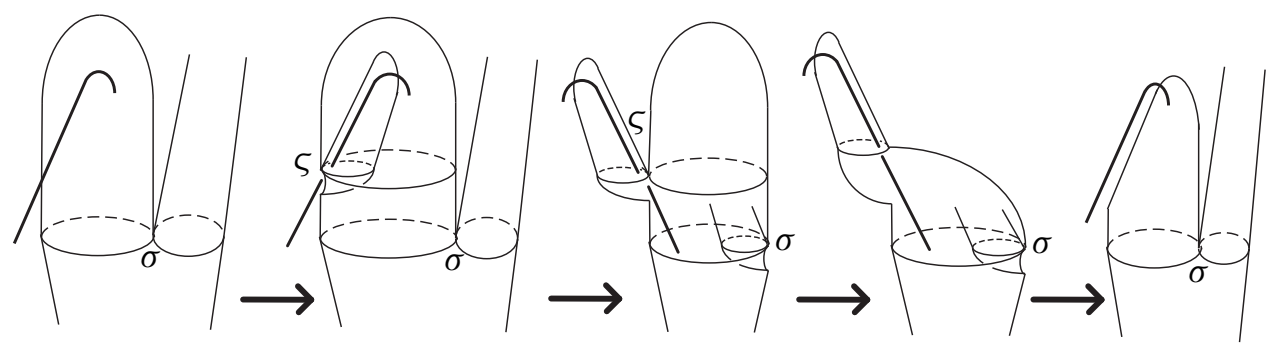

Figure 5

of maxima of $\left.h\right|_{K}$, but replaces $D_{\sigma}$ with a different outermost disk so that $\sigma$ is now a removable c-inessential saddle. By Lemma 3.5, $C$ is not taut.

The sphere $C$ decomposes $S^{3}$ into two 3 -balls $B_{1}$ and $B_{2}$. Let $\sigma$ be a saddle in $F_{C}$ and $L$ be the level sphere either just above or just below $\sigma$ that contains $c_{1}^{\sigma}$ and $c_{2}^{\sigma}$. $L-\left(c_{1}^{\sigma} \cup c_{2}^{\sigma}\right)$ is composed of two disks and an annulus $A$. If a collar of $\partial(A)$ in $A$ is contained in $B_{1}$, then we say $\sigma$ is unnested with respect to $B_{1}$. If not, we say $\sigma$ is nested with respect to $B_{1}$. We define nested and unnested with respect to $B_{2}$ similarly. Note that nested with respect to $B_{1}$ is the same as unnested with respect to $B_{2}$ and nested with respect to $B_{2}$ is unnested with respect to $B_{1}$.

Two saddles $\sigma=s_{1}^{\sigma} \vee s_{2}^{\sigma}$ and $\tau=s_{1}^{\tau} \vee s_{2}^{\tau}$ in $F_{C}$ are adjacent if, up to labeling, $s_{1}^{\sigma}$ and $s_{1}^{\tau}$ cobound an annulus in $C$ that is disjoint from $s_{2}^{\sigma}, s_{2}^{\tau}$, all other saddles, and $K$. Recall that, if $\sigma$ is a standard saddle, $E_{\sigma}$ is the monotone disk with boundary $\sigma$.

Lemma 3.7 If $\sigma$ and $\tau$ are adjacent saddles with $\sigma$ a standard saddle such that $E_{\sigma} \cap K=\varnothing$ and $\sigma$ and $\tau$ are nested with respect to different 3-balls, then $C$ is not taut.

Proof This is Schultens' Lemma 3 in [6].

\section{Thin position, pods and nesting}

In this section, we will exploit a connection between the existence of c-inessential saddles and an isotopy which thins $K$. Marty Scharlemann coined the term "Pods" for the arrangement of c-inessential saddles that give rise to this isotopy.

Following [3], we make the following definitions. 
Definition 4.1 Suppose $K \subset S^{3}$ is in general position with respect to the standard height function $h, c_{0}<c_{1}<\cdots<c_{n}$ are the critical values of $\left.h\right|_{K}$ and the regular values $r_{i}$ are chosen so that $c_{i-1}<r_{i}<c_{i}, i=1, \ldots, n$. The width of $K$ with respect to $h$, denoted by $w(K, h)$, is $\sum_{i}\left|h^{-1}\left(r_{i}\right) \cap K\right|$. The width of $K$, denoted by $w(K)$, is the minimum of $w\left(K^{\prime}, h\right)$ over all knots $K^{\prime}$ isotopic to $K$. We say that $K$ is in thin position if $w(K, h)=w(K)$.

Recall the definition of bridge position from Section 2. We will be making extensive use of the following fact: If $\left.h\right|_{K}$ has $\beta(K)$ maxima and there is a minimum of $\left.h\right|_{K}$ above a maximum of $\left.h\right|_{K}$, then bridge position for $K$ is not thin position for $K$.

Definition 4.2 A surface $F$ in $S^{3}-K$ is c-incompressible if every disk or 1-punctured disk $D$ in $S^{3}-K$ with $D \cap F=\partial(D)$ is properly isotopic into $F$.

In particular, we will be analyzing c-incompressible Conway spheres. A Conway sphere $C$ is c-incompressible in the complement of a knot $K$ if and only if $C$ is incompressible and neither complementary tangle contains a summand of $K$. Hence, if $K$ is prime, then every incompressible Conway sphere is c-incompressible.

Recall that if $\sigma$ is a c-inessential saddle, then $B_{\sigma}$ is the 3-ball with boundary $D_{\sigma} \cup D_{1}^{\sigma}$ and $B_{\sigma}^{\prime}$ is the 3-ball with boundary $D_{\sigma} \cup\left(S_{\sigma}-D_{1}^{\sigma}\right)$ where $S_{\sigma}$ is the level surface containing $\sigma$. See Figure 1 .

Lemma 4.3 If $C$ is a taut c-incompressible Conway sphere in $S^{3}-K$ and $\sigma$ is a $c$-inessential saddle in $F_{C}$ such that $D_{\sigma}$ contains a maximum (minimum) of $\left.h\right|_{C}$, then each of $B_{\sigma}$ and $B_{\sigma}^{\prime}$ contain a maximum (minimum) of $\left.h\right|_{K}$.

Proof Without loss of generality we can assume $D_{\sigma}$ contains a maximum. Since $C$ is taut, $\sigma$ is nonremovable, by Lemma 3.5. Since $\sigma$ is nonremovable, $\left.h\right|_{K \cap B_{\sigma}}$ has a local minimum at $p_{\sigma}=\left.K \cap D_{\sigma} \cdot h\right|_{K}$ is initially increasing as $K$ passes into $B_{\sigma}$ and subsequently decreases to exit $B_{\sigma}$ through $D_{1}^{\sigma}$. Hence, $\left.h\right|_{K}$ must have a maximum in $B_{\sigma}$.

If $K \cap B_{\sigma}^{\prime}$ is not a monotone arc, then there is a maximum of $\left.h\right|_{K}$ in $B_{\sigma}^{\prime}$ and we are done.

Suppose $K \cap B_{\sigma}^{\prime}$ is a monotone arc with the lower endpoint not in $D_{2}^{\sigma}$, then $D_{2}^{\sigma} \cap K=$ $\varnothing$. $C \cap D_{2}^{\sigma}$ is a collection of disjoint simple closed curves. An innermost such curve $\alpha$ bounds a disk $E_{1}$ in $D_{2}^{\sigma}$ such that $E_{1}$ is disjoint from $K$ and $E_{1} \cap C=\partial\left(E_{1}\right)$. By c-incompressibility of $C, \alpha$ also bounds a disk $E_{2}$ in $C$ that is disjoint from $K$. Additionally, $E_{1}$ and $E_{2}$ cobound a 3 -ball that is disjoint from $K$, since $K$ is a 
nonsplit link. See Figure 6. Hence, we can eliminate $\alpha$ as a curve of intersection by an isotopy which takes $E_{2}$ across this 3 -ball and just past $E_{1}$. This isotopy leaves $K$ fixed and can only decrease the number of saddles in $F_{C}$. By repeating this process, we can eliminate all curves of intersection of $C$ with $\operatorname{int}\left(D_{2}^{\sigma}\right)$. Again, by c-incompressibility of $C, s_{2}^{\sigma}$ bounds a disk $E_{2}$ in $C$ which is disjoint from $K$. So, $\sigma$ is an inessential saddle or an innermost saddle of $F_{E_{2}}$ is an inessential saddle. By Lemma 3.3, the existence of an inessential saddle implies $C$ is not taut.

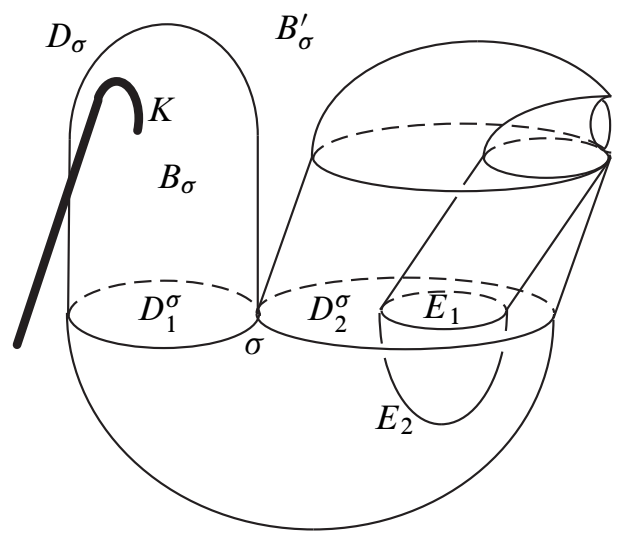

Figure 6

Suppose $K \cap B_{\sigma}^{\prime}$ is a monotone arc with the lower endpoint in $D_{2}^{\sigma}$. Let $L$ be the level surface containing $\sigma$. Since $K \cap B_{\sigma}^{\prime}$ is a monotone arc, $K$ meets $L$ exactly once outside of $D_{1}^{\sigma}$. Hence, $K \cap D_{2}^{\sigma}$ consists of a single point. $C \cap D_{2}^{\sigma}$ is a collection of disjoint simple close curves. An innermost such curve $\alpha$ bounds a disk $E_{1}$ in $D_{2}^{\sigma}$ that is disjoint from $C$ except in its boundary. Since $K$ meets all of $D_{2}^{\sigma}$ in a single point, $K$ meets $E_{1}$ in at most one point. See Figure 7. If $E_{1} \cap K=\varnothing$, then, as described in the above paragraph, there is an isotopy of $C$ which eliminates $\alpha$, leaves $K$ fixed, and can only decrease the number of saddles in $F_{C}$. If $E_{1} \cap K$ is a single point, then, by c-incompressibility of $C, \alpha$ bounds a 1-punctured disk $E_{2}$ in $C$ such that $E_{1} \cup E_{2}$ bounds a 3-ball containing a single unknotted subarc of $K$. Hence, we can eliminate $\alpha$ as an arc of intersection by an isotopy which takes $E_{2}$ across this 3-ball and just past $E_{1}$. This isotopy leaves $K$ fixed and can only decrease the number of saddles in $F_{C}$. By repeating this process, we can eliminate all arcs of intersection of $C$ with $\operatorname{int}\left(D_{2}^{\sigma}\right)$. Again, by c-incompressibility of $C, s_{2}^{\sigma}$ bounds a 1-punctured disk $E_{2}$ in $C$ such that $D_{2}^{\sigma} \cup E_{2}$ bounds a 3-ball containing a single unknotted subarc of $K$. We can isotope $E_{2}$ to $D_{2}^{\sigma}$ across this 3-ball leaving $K$ fixed. After a small tilt, this isotopy results in a new Conway sphere in general position with respect to $h$ but with 
one fewer saddle, having eliminated $\sigma$. Since $K$ was fixed at every step of the isotopy, we have shown the original $C$ is not taut with respect to $\beta(K)$.

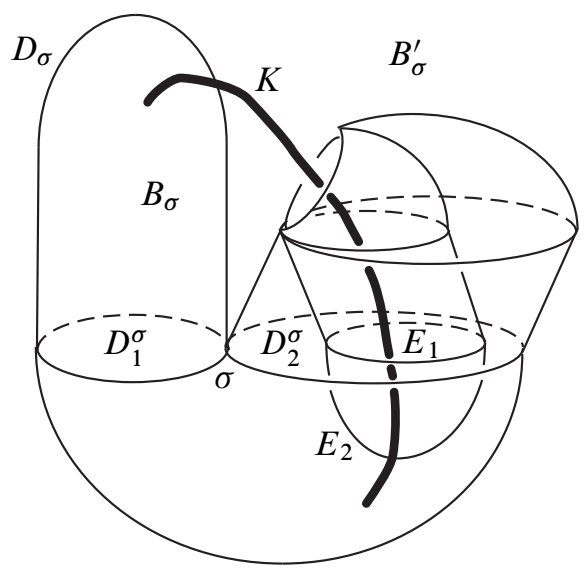

Figure 7

Recall that $\gamma_{\sigma}$ is the monotone subarc of $K$ that meets $D_{\sigma}$ for some c-inessential saddle $\sigma$ in $F_{C}$.

Definition 4.4 We say a c-inessential saddle $\sigma$ in $F_{C}$ is a pod if $D_{\sigma}$ contains a maximum (minimum), there is an additional c-inessential saddle $\varsigma$ such that $D_{\zeta} \cap K$ is a point on $\gamma_{\sigma}$ above(below) $D_{\sigma} \cap K$, and $D_{\varsigma}$ has a unique minimum(maximum). See Figure 8.

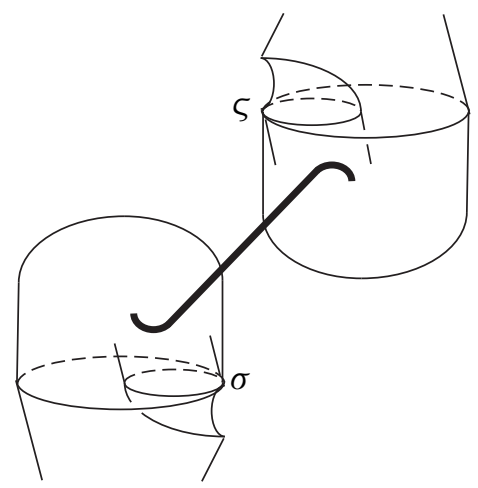

Figure 8 
Lemma 4.5 If $C$ is a taut c-incompressible Conway sphere in $S^{3}-K$ and $\sigma$ is a pod, then bridge position for $K$ is not thin position.

Proof Without loss of generality, suppose $D_{\sigma}$ contains a maximum. Let $\gamma_{\sigma} \cap D_{\sigma}=a$ and $\gamma_{\sigma} \cap D_{\varsigma}=b$, where $\varsigma$ is a c-inessential saddle, and $h(a)<h(b)$. By Lemma 3.5, we can assume both $\sigma$ and $\varsigma$ are nonremovable saddles. Let $S$ be the level surface containing $\varsigma$. By the definition of pod, $D_{\varsigma}$ has a unique minimum. $D_{\varsigma}$ divides the 3ball below $S$ into two 3-balls $B_{\varsigma}$ and $B_{\varsigma}^{\prime}$. Since $\varsigma$ is nonremovable, $B_{\varsigma}$ contains $\sigma$. Since $\sigma$ and $\varsigma$ are nonremovable, c-inessential saddles in $F_{C}$ such that $D_{\sigma}$ contains a maximum and $D_{\varsigma}$ contains a minimum, then $h_{K \cap B_{\sigma}^{\prime}}$ has a local maximum at $K \cap D_{\sigma}$ and $h_{K \cap B_{\varsigma}^{\prime}}$ has a local minimum at $K \cap D_{\varsigma}$. We can now appeal to the isotopy in Lemma 3.5 to horizontally shrink and vertically lower $B_{\sigma}^{\prime}$ and horizontally shrink and vertically lift $B_{\varsigma}^{\prime}$. Since $h\left(D_{\sigma} \cap K\right)<h\left(D_{\varsigma} \cap K\right)$, then $h(\sigma)<h(\varsigma)$. Hence, we can lower $B_{\sigma}^{\prime}$ and lift $B_{\varsigma}^{\prime}$ until $B_{\sigma}^{\prime}$ lies strictly below $B_{\varsigma}^{\prime}$. However, by Lemma 4.3, $B_{\sigma}^{\prime}$ contains a maximum of $\left.h\right|_{K}$ and $B_{\varsigma}^{\prime}$ contains a minimum of $\left.h\right|_{K}$. See Figure 9. Thus, bridge position for $K$ is not thin position for $K$.

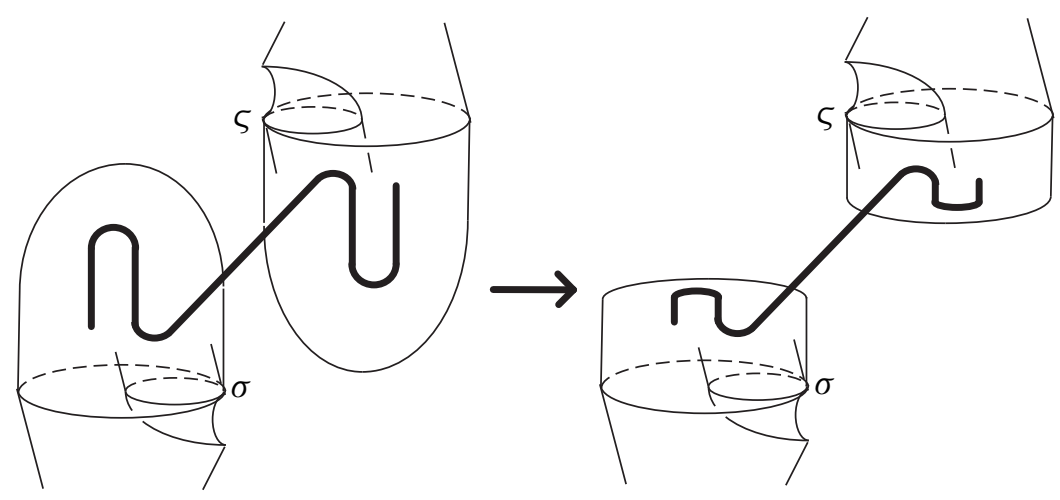

Figure 9

Recall the definitions of standard, outermost, $B_{\sigma}$ and $A_{\sigma}$, from Section 2.

Lemma 4.6 If $C$ is a taut Conway sphere in $S^{3}-K$ and $\sigma$ is an outermost standard saddle in $F_{C}$ such that $B_{\sigma} \cap C \neq \varnothing$, then $\left(B_{\sigma} \cup A_{\sigma}\right)^{c}$ contains a nonstandard saddle of $F_{C}$ or a point of $K \cap C$.

Proof Assume $\sigma$ is nested with respect to $B_{1}$. Since $C$ is connected and $B_{\sigma} \cap C \neq \varnothing$, then $\left(B_{\sigma} \cup A_{\sigma}\right)^{c}$ contains at least one saddle. Let $\tau$ be the saddle in $\left(B_{\sigma} \cup A_{\sigma}\right)^{c}$ nearest $s_{2}^{\sigma}$ in $C$. If $\tau$ is nonstandard then we are done. In particular, we can assume 
$s_{2}^{\sigma}$ cobounds a monotone annulus $A^{\tau}$ with $s_{1}^{\tau}$. If $\tau$ is nested with respect to $B_{2}$ and $\left(A^{\tau} \cup E_{\tau}\right) \cap K=\varnothing$, then, by Lemma 3.7, we contradict $C$ being taut. If $\left(A^{\tau} \cup E_{\tau}\right) \cap K \neq \varnothing$, then we have a point of $K \cap C$ in $\left(B_{\sigma} \cup A_{\sigma}\right)^{c}$ and we are done. Hence, we can assume $\tau$ is a standard saddle nested with respect to $B_{1} . s_{2}^{\tau}$ can not bound an outermost disk since $B_{\sigma} \cap C \neq \varnothing$. Let $\varsigma$ be the saddle nearest to $\tau$ in $F_{C}$ in the direction of $c_{2}^{\tau}$. Since $\tau$ is nested with respect to $B_{1}, \varsigma$ must be in $\left(B_{\sigma} \cup A_{\sigma}\right)^{c}$. If $\varsigma$ is nonstandard we are done. Hence, we can assume that $s_{2}^{\tau}$ and $s_{1}^{\varsigma}$ cobound a monotone annulus. We repeat the above argumentation to conclude $\varsigma$ is nested with respect to $B_{1}$ or there is a point of $K \cap C$ in $\left(B_{\sigma} \cup A_{\sigma}\right)^{c}$. Inductively, all finitely-many saddles of $F_{C}$ are standard and nested with respect to $B_{1}$ or there is a point of $K \cap C$ in $\left(B_{\sigma} \cup A_{\sigma}\right)^{c}$. However, all saddles in $F_{C}$ being standard and nested with respect to $B_{1}$ contradicts $B_{\sigma} \cap C \neq \varnothing$. Hence, $\left(B_{\sigma} \cup A_{\sigma}\right)^{c}$ contains a nonstandard saddle of $F_{C}$ or a point of $K \cap C$.

\section{Nonremovable saddles}

Lemma 5.1 The number of outermost disks in $F_{C}$ is two more than the number of nonstandard saddles in $F_{C}$.

Proof Let $\mathcal{A}$ be the collection of all curves $c_{\sigma}^{i}$ where $i \epsilon\{1,2,3\}$ and $\sigma$ is any saddle in $F_{C}$. Viewing $C$ as an embedded surface in $S^{3}, \mathcal{A}$ decomposes $C$ into $x$ monotone disks, $y$ pairs of pants and $z$ vertical annuli. See Figure 10. Hence, $\chi(C)=2=x-y$. Let $x_{1}$ be the number of monotone disks in $C-\mathcal{A}$ with boundary $c_{\sigma}^{1}$ or $c_{\sigma}^{2}$ for some saddle $\sigma$. Let $x_{2}$ be the number of monotone disks in $C-\mathcal{A}$ with boundary $c_{\sigma}^{3}$ for some saddle $\sigma$. By the definition of standard saddle, $x_{2}$ is the number of standard saddles in $F_{C}$ and $y-x_{2}$ is the number of nonstandard saddles in $F_{C}$. By definition of outermost disk, $x_{1}$ is the number of outermost disks. Since $2=x-y$ and $x=x_{1}+x_{2}$, then $x_{1}=\left(y-x_{2}\right)+2$. Hence, The number of outermost disks in $F_{C}$ is two more than the number of nonstandard saddles in $F_{C}$.

Remark 1 If there are more than four outermost disks in $F_{C}$, then one of these disks does not meet $K$, so, there is an inessential saddle in $F_{C}$. By Lemma 3.3, if $C$ is taut, then $F_{C}$ contains four or fewer outermost disks and two or fewer nonstandard saddles. Similarly, for every point in $K \cap C$ that is not contained in the collection of outermost disks of $F_{C}$ there is one fewer outermost disk and one fewer nonstandard saddle possible in $F_{C}$.

Definition 5.2 We say a saddle $\sigma$ in $F_{C}$ is doubly c-inessential if both $s_{1}^{\sigma}$ and $s_{2}^{\sigma}$ bound outermost disks $D_{\sigma}$ and $D_{\sigma}^{\prime}$ in $F_{C}$ such that each of $D_{\sigma}$ and $D_{\sigma}^{\prime}$ meet $K$ in exactly one point. 


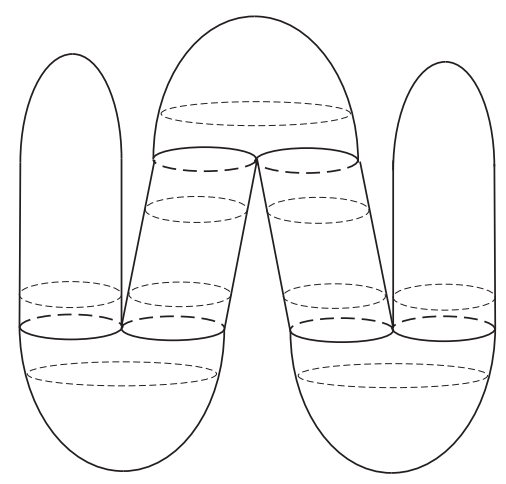

Figure 10

Lemma 5.3 If $C$ is a taut c-incompressible Conway sphere and bridge position for $K$ is thin position then $F_{C}$ does not contain any doubly c-inessential saddles.

Proof Suppose $\sigma$ is a doubly c-inessential saddle. Assume that both $D_{\sigma}$ and $D_{\sigma}^{\prime}$ have maxima (the case where $D_{\sigma}$ and $D_{\sigma}^{\prime}$ have minima is proved similarly). Let $B_{\sigma}$ be the 3-ball disjoint from $s_{2}^{\sigma}$ with boundary $D_{\sigma} \cup D_{1}^{\sigma}$. Similarly, let $B_{\sigma}^{\prime}$ be the 3-ball disjoint from $s_{2}^{\sigma}$ with boundary $D_{\sigma}^{\prime} \cup D_{2}^{\sigma}$. If $\left.h\right|_{K \cap B_{\sigma}}$ has a maximum at $K \cap D_{\sigma}$ or $\left.h\right|_{K \cap B_{\sigma}^{\prime}}$ has a maximum at $K \cap D_{\sigma}^{\prime}$, then $\sigma$ is a removable saddle and $C$ is not taut, by Lemma 3.5. Let $\gamma_{\sigma}$ be the monotone strand of $K$ that meets $D_{\sigma}$ and $\gamma_{\sigma}^{\prime}$ be the monotone strand of $K$ that meets $D_{\sigma}^{\prime}$. If $\gamma_{\sigma}$ is disjoint from $C$ above $K \cap D_{\sigma}$ or $\gamma_{\sigma}^{\prime}$ is disjoint from $C$ above $K \cap D_{\sigma}^{\prime}$, then $F_{C}$ is not taut, by Lemma 3.6. Hence, $C$ meets both the interior of $B_{\sigma}$ and the interior of $B_{\sigma}^{\prime}$. Since $C \cap B_{\sigma} \neq \varnothing$, then $\sigma$ must be a nonstandard saddle. Let $x_{1}$ be the point of $K \cap C$ above $K \cap D_{\sigma}$ on $\gamma_{\sigma}$ and $x_{2}$ be the point of $K \cap C$ above $K \cap D_{\sigma}^{\prime}$ on $\gamma_{\sigma}^{\prime}$. By Remark 1, $\sigma$ nonstandard implies at least one of $x_{1}$ or $x_{2}$ lies on an outermost disk of $F_{C} \cdot x_{1}$ and $x_{2}$ cannot lie on a common monotone disk of $F_{C}$ since such a disk cannot meet both the interior of $B_{\sigma}$ and the interior of $B_{\sigma}^{\prime}$. Figure 11 illustrates one potential embedding of $C$.

Without loss of generality, suppose $x_{1}$ lies on an outermost disk $D_{\varsigma}$ of $F_{C} \cdot x_{1}$ must be the unique point of $C \cap K$ that meets $D_{\varsigma}$, so $\varsigma$ is a c-inessential saddle.

If $\varsigma$ is a removable c-inessential saddle, then, by Lemma 3.5, $C$ is not taut.

If $\varsigma$ is a nonremovable c-inessential saddle such that $D_{\varsigma}$ has a minimum, then $\varsigma$ and $\sigma$ are pods. By Lemma 4.5, $C$ is not taut or bridge position for $K$ is not thin position.

If $\varsigma$ is a nonremovable c-inessential saddle such that $D_{\varsigma}$ has a maximum, then $\gamma_{\varsigma}$ is disjoint from $C$ above $x_{1}$ and, by Lemma 3.6, $C$ is not taut. 


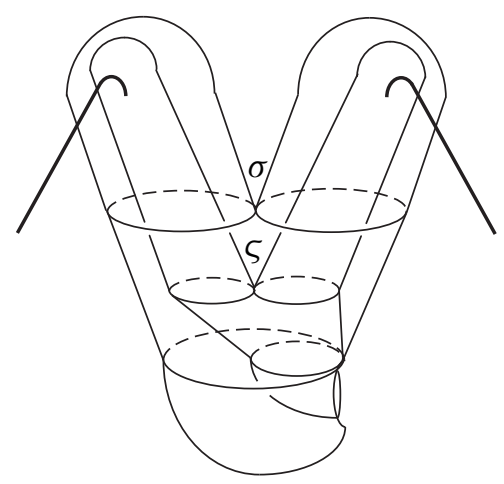

Figure 11

Corollary 5.4 If $C$ is a taut c-incompressible Conway sphere in $S^{3}-K$ and bridge position for $K$ is thin position, then there is a one-to-one correspondence between nonremovable c-inessential saddles of $F_{C}$ and outermost disks of $F_{C}$ that meet $K$ in exactly one point.

Proof There is an obvious well-defined map between 1-punctured outermost disks and c-inessential saddles. This map sends each outermost disk to the saddle that contains its boundary. If two or more disks get mapped to the same saddle then this saddle is doubly c-inessential. However, this contradicts Lemma 5.3. Thus, there is a one-to-one correspondence between nonremovable c-inessential saddles of $F_{C}$ and outermost disks of $F_{C}$ that meet $K$ in exactly one point.

Lemma 5.5 Let $C$ be a c-incompressible Conway sphere in $S^{3}-K$. If $F_{C}$ contains distinct $c$-inessential saddles $\sigma$ and $\varsigma$ such that $D_{\sigma}$ has a maximum (minimum) and $D_{\varsigma}$ meets $\gamma_{\sigma}$ above(below) $D_{\sigma} \cap K$, then $C$ is not taut or bridge position for $K$ is not thin position.

Proof Both $\sigma$ and $\varsigma$ are nonremovable or else $C$ is not taut, by Lemma 3.5. Without loss of generality, suppose $D_{\sigma}$ contains a maximum and $D_{\varsigma} \cap K=b$ is above $D_{\sigma} \cap K=a$ on $\gamma_{\sigma}$. If $D_{\varsigma}$ has a minimum, then $\sigma$ is a pod and $C$ is not taut or bridge position for $K$ is not thin position, by Lemma 4.5. Hence, we can assume that $D_{\varsigma}$ has a maximum.

Suppose $C$ meets $\gamma_{\sigma}$ exactly once above $a$. Then $C$ is disjoint from $\gamma_{\sigma}$ above $b$. By Lemma 3.6, $C$ is not taut.

Suppose $C$ meets $\gamma_{\sigma}$ exactly twice above $a$ in the points $b$ and $c$. If $b$ is the highest of these two points, then, by Lemma 3.6, $C$ is not taut. If $c$ is the highest of these two 
points, poke a small neighborhood of the point $c$ in $C$ along $\gamma_{\sigma}$ toward and slightly past the maximum of $\gamma_{\sigma}$ as in the proof of Lemma 3.6. This isotopy fixes $K$, but adds a single c-inessential saddle to $F_{C}$. After this isotopy, $\{a, b\}=\gamma_{\sigma} \cap C$. Hence, we can perform the isotopy in Lemma 3.6 to eliminate first $\varsigma$ and subsequently $\sigma$. See Figure 12. The net effect of this isotopy is to decrease the number of saddles in $F_{C}$ by one while preserving the number of maxima of $\left.h\right|_{K}$. This contradicts the assumption that $C$ is taut.
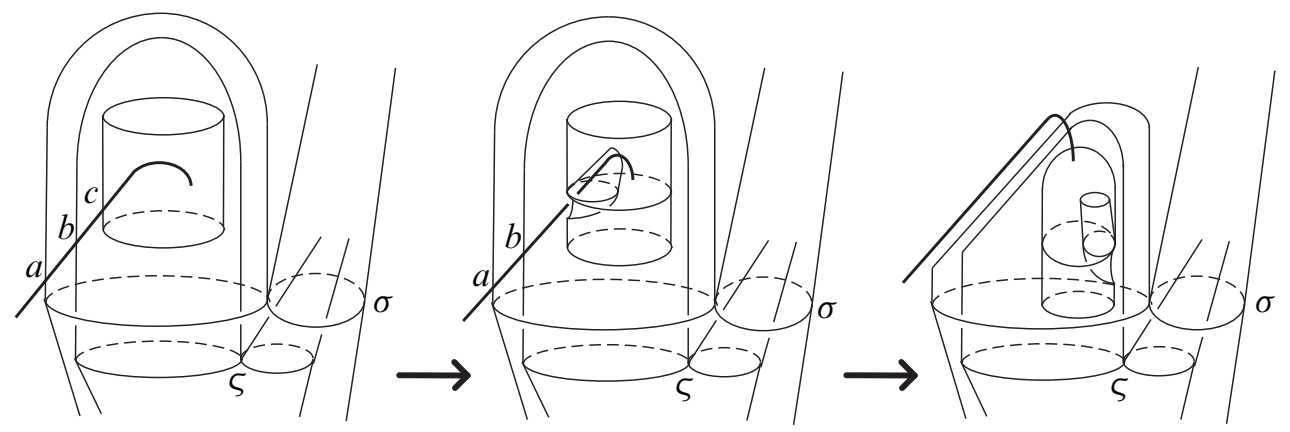

Figure 12

Suppose $C$ meets $\gamma_{\sigma}$ exactly three times above $D_{\sigma} \cap K$ in points $b, c$ and $d$. In this case all points of $K \cap C$ are contained in $B_{\sigma}$. By Lemma 4.6, either $\sigma$ is nonstandard or there exists a nonstandard saddle of $F_{C}$ in $\left(B_{\sigma} \cup A_{\sigma}\right)^{c}$. Then, by Remark 1, there are at least three outermost disks $D_{\sigma}, D_{\zeta}$, and $D_{\tau}$ in $F_{C}$. Again by Remark 1, at least one of $D_{\varsigma}$ and $D_{\tau}$ meet $K$ in a single point. We now analyze the possibilities for $D_{\tau}$.

Assume $D_{\tau}$ meets $K$ exactly once at the point $c . \tau$ is a nonremovable c-inessential saddle or else $C$ is not taut, by Lemma 3.5. If $D_{\tau}$ has a minimum, then $\tau$ and $\sigma$ are pods and, by Lemma 4.5, $C$ is not taut or bridge position for $K$ is not thin position. So, we can assume $D_{\tau}$ has a maximum. Recall from the first paragraph of this proof that we have assumed $D_{\varsigma}$ has a maximum.

If $b$ or $c$ is the highest of the four points on $\gamma_{\sigma}$, we can use the isotopy from Lemma 3.6 to eliminate $\varsigma$ or $\tau$ contradicting the fact that $C$ is taut. Hence, we can assume $d$ is that highest of the four points. Poke a small neighborhood of the point $d$ in $C$ in along $\gamma_{\sigma}$ toward and slightly past the maximum of $\gamma_{\sigma}$. This isotopy fixes $K$, but adds a single c-inessential saddle to $F_{C}$. After this isotopy, $\{a, b, c\}=\gamma_{\sigma} \cap C$. Hence, we can perform the isotopy in Lemma 3.6 to eliminate first $\tau$, then $\varsigma$ and finally $\sigma$. This isotopy is similar to that of Figure 12, but with three nonremovable, c-inessential saddles. The net effect of this isotopy is to decrease the number of saddles in $F_{C}$ by 
two while preserving the number of maxima of $\left.h\right|_{K}$. Hence, we conclude $C$ is not taut.

Assume $D_{\tau}$ meets $K$ exactly twice. Label the points of $\gamma_{\sigma} \cap C$ from lowest to highest as $\{a, b, c, d\}$ where $a=K \cap D_{\sigma}$. If $d=K \cap D_{\varsigma}$, then we can eliminate $\varsigma$ as in Lemma 3.6 and conclude $F_{C}$ is not taut. By parity, the labels of the two points in $K \cap D_{\tau}$ must be consecutive. Hence, $b=K \cap D_{\varsigma}$ and $\{c, d\}=K \cap D_{\tau}$.

Suppose $D_{\tau}$ contains a maximum. Poke a small neighborhood of $d$ in $D_{\tau}$ along $\gamma_{\sigma}$ toward and just past the maximum of $\gamma_{\sigma}$. Since $\gamma_{\sigma}$ is disjoint from $C$ above $d$, this isotopy creates a single new inessential saddle. Eliminate this saddle using the isotopy in Lemma 3.3. After these isotopies, $\{a, b, c\}=C \cap \gamma_{\sigma}$. Poke a small neighborhood of the point $c$ in $C$ in along $\gamma_{\sigma}$ toward and slightly past the maximum of $\gamma_{\sigma}$. This isotopy fixes $K$ but adds a single c-inessential saddle to $F_{C}$ as in Figure 13. After this isotopy, $\{a, b\}=\gamma_{\sigma} \cap C$.

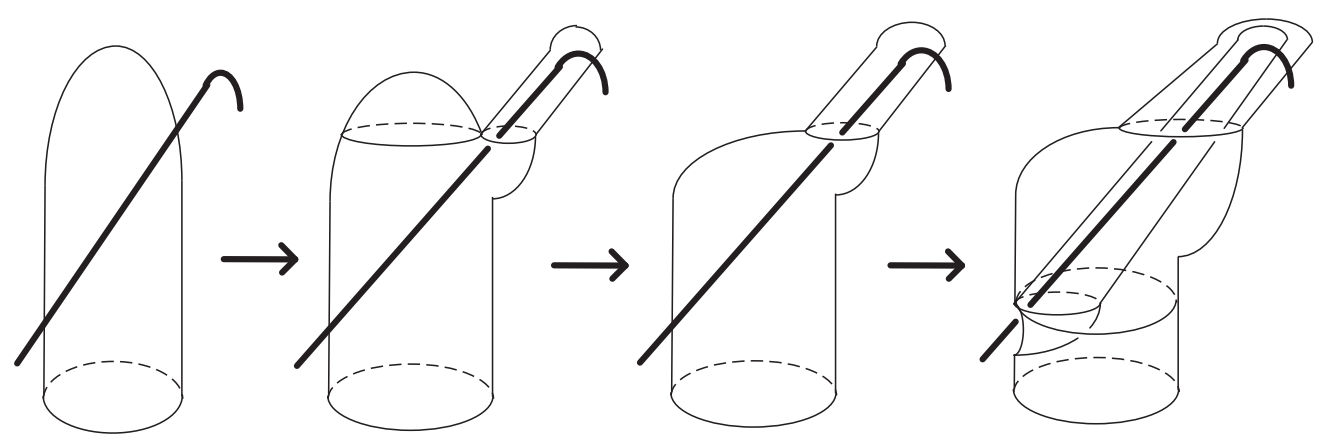

Figure 13

Hence, we can perform the isotopy in Lemma 3.6 to eliminate first $\varsigma$ and then $\sigma$. The net effect of this isotopy is to decrease the number of saddles in $F_{C}$ by one while preserving the number of maxima of $\left.h\right|_{K}$. Hence, $C$ is not taut.

Suppose $D_{\tau}$ contains a minimum. Let $S_{\tau}$ be the level surface containing $\tau . D_{\tau}$ divides the 3-ball below $S_{\tau}$ into two three balls $B_{\tau}$ and $B_{\tau}^{\prime}$ where we choose the labels in the unconventional way where $B_{\tau}$ contains $\sigma$ and may or may not contain $s_{2}^{\tau}$ in its boundary.

Let $F$ be a level disk properly embedded in $B_{\tau}^{\prime}$ that lies below $\tau$ but above $d$.

Claim There is a minimum of $\left.h\right|_{K}$ contained in $B_{\tau}^{\prime}$ below $F$. 
Proof We know by hypothesis that there is a monotone subarc of $K$ in $B_{\tau}^{\prime}$ connecting $c$ to $d$. Suppose, to form a contradiction, that $F \cap K=\varnothing . C \cap F$ is a collection of disjoint simple close curves. An innermost such curve $\alpha$ bounds a disk $E_{1}$ in $F$ with interior disjoint from both $C$ and $K$. By c-incompressibility of $C, \alpha$ also bounds a disk $E_{2}$ in $C$ that is disjoint from $K$. Additionally, $E_{1}$ and $E_{2}$ cobound a 3-ball which is disjoint from $K$, since $K$ is not split. Hence, we can eliminate $\alpha$ as an arc of intersection by an isotopy which takes $E_{2}$ across this 3-ball and just past $E_{1}$. This isotopy leaves $K$ fixed and can only decrease the number of saddles in $F_{C}$. By repeating this process, we can eliminate all curves of intersection of $C$ with $\operatorname{int}(F)$. If $C$ and $K$ are disjoint from $\operatorname{int}(F)$, then $F$ is an essential compressing disk for $C$ and we contradict c-incompressibility of $C$. We conclude that $K \cap F \neq \varnothing$. However, any arc of $K$ with endpoints in $F$ must have a minimum in $B_{\tau}^{\prime}$ below $F$. This proves the claim.

By appealing to Lemma 3.2, we can assume $B_{\sigma}^{\prime}$ does not contain $+\infty$ and $B_{\tau}^{\prime}$ does not contain $-\infty$. Horizontally shrink and vertically lift the portion of $B_{\tau}^{\prime}$ below $c$ until the minimum of $D_{\tau}$ is just below $c$. Horizontally shrink and vertically lower $B_{\sigma}^{\prime}$ until the maximum of $D_{\sigma}$ is just above $a$. Since $h(c)>h(a)$, this isotopy insures that $B_{\tau}^{\prime}$ lies completely above $B_{\sigma}^{\prime}$. By Lemma 4.3 , there is a maximum of $\left.h\right|_{K}$ in $B_{\sigma}^{\prime}$ and, by the above claim, there is a minimum of $\left.h\right|_{K}$ in $B_{\tau}^{\prime}$. Bridge position for $K$ is not thin position for $K$ since we have isotoped a minimum of $\left.h\right|_{K}$ above a maximum of $\left.h\right|_{K}$ without introducing any new critical points. See Figure 14.
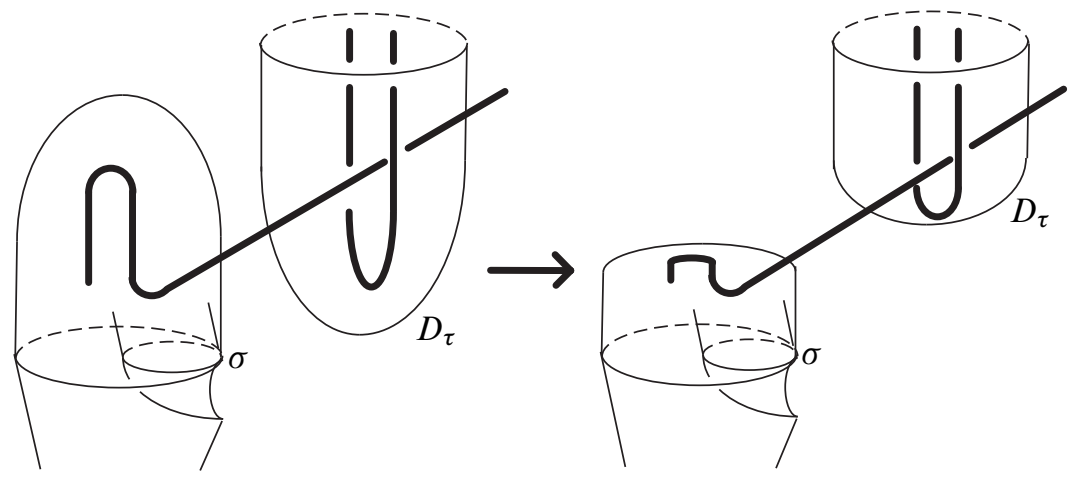

Figure 14

Theorem 5.6 Let $C$ be a c-incompressible Conway sphere in $S^{3}-K$. If $F_{C}$ contains both a c-inessential saddle $\sigma$ and a nonstandard saddle, then $C$ is not taut or bridge position for $K$ is not thin position. 
Proof If $\sigma$ is removable then $C$ is not taut, by Lemma 3.5. Hence we can assume $\sigma$ is nonremovable and $D_{\sigma}$ has a unique maximum. By Remark $1, F_{C}$ contains at least three outermost disks with each disk meeting $K$ at least once or $C$ is not taut. Hence, we will assume $F_{C}$ contains a second c-inessential saddle $\tau$. We will break the proof up into cases based on $\left|C \cap \gamma_{\sigma}\right|$.

Suppose $\left|C \cap \gamma_{\sigma}\right|=1$. By Lemma 3.6, $C$ is not taut.

Suppose $\left|C \cap \gamma_{\sigma}\right|=2$. Let $\{a, b\}=C \cap \gamma_{\sigma}$ where $a=D_{\sigma} \cap K$. By Lemma 3.6, $h(b)<h(a)$ implies $C$ is not taut. Assume $h(b)>h(a)$ and we proceed by cases.

Case I Suppose $b \in D_{\varsigma}$ for some c-inessential saddle $\varsigma$ in $F_{C}$. By Lemma 5.5, $C$ is not taut or bridge position for $K$ is not thin position.

Case II Suppose $b$ is not the puncture associated to a c-inessential saddle of $F_{C}$.

Case II.A Additionally, suppose that $b$ is not contained in an outermost disk in $F_{C}$. Since $b$ is not contained in an outermost disk and $C$ is taut, then each of the three outermost disks of $F_{C}$ meets $K$ exactly once. By Corollary 5.4, $F_{C}$ contains three distinct c-inessential saddles, $\sigma, \varsigma$ and $\tau$. By Lemma 3.5, we can assume both $\varsigma$ and $\tau$ are nonremovable. Assume that $D_{\varsigma}$ has a unique maximum. By Lemma 3.6, $C$ meets $\gamma_{\varsigma}$ above $D_{\varsigma} \cap K$ or $C$ is not taut. Since $\left|C \cap \gamma_{\sigma}\right|=2$ and $b \neq D_{\varsigma} \cap K$, then $\gamma_{\varsigma}$ is distinct from $\gamma_{\sigma}$ and $D_{\tau} \cap K$ is the unique point of intersection of $C$ with $\gamma_{\varsigma}$ above $D_{\varsigma} \cap K$. By Lemma 5.5, $C$ is not taut or bridge position for $K$ is not thin position. The proof when $D_{\varsigma}$ has a unique minimum follows similarly.

Case II.B Suppose $b$ is contained in an outermost disk $D_{\varsigma}$ where $\partial\left(D_{\varsigma}\right)=s_{1}^{\varsigma}$ for some saddle $\varsigma=s_{1}^{\varsigma} \vee s_{2}^{\varsigma}$ in $F_{C}$. Since we have assumed $b$ is not the puncture associated to a c-inessential saddle of $F_{C}$, then $D_{\varsigma}$ must have one or two additional punctures. Since each of the three outermost disks must meet $K$ at least once, $D_{\varsigma}$ meets $K$ exactly twice. Let $D_{\varsigma} \cap K=\left\{b, b^{\prime}\right\}$.

The other c-inessential saddle $\tau$, like $\sigma$, must be nonremovable and $\left|\gamma_{\tau} \cap C\right| \geq 2$ or else we can use Lemma 3.5 or Lemma 3.6 to show $C$ is not taut. By assumption, $\left|C \cap \gamma_{\sigma}\right|=2$ and, thus, $\gamma_{\sigma}$ is distinct from $\gamma_{\tau}$.

Hence, $C \cap \gamma_{\tau}=\left\{c, b^{\prime}\right\}$ where $c=D_{\tau} \cap K$. We have now accounted for all four points of $K \cap C$ ( $c$ and $b^{\prime}$ on $\gamma_{\tau}$, and $a$ and $b$ on $\gamma_{\sigma}$ ). We focus on the monotone strand of $K$ that meets $D_{\varsigma}$ nearest its maxima (minima). If this strand is $\gamma_{\sigma}$, then our labeling stays the same and we move on. If this strand is $\gamma_{\tau}$, then we swap the labels $b$ and $b^{\prime}$ as well as $\tau$ and $\sigma$. Additionally, we reflect along a level sphere if necessary so that $D_{\sigma}$ continues to have a unique maximum. Figure 15 illustrates one possible configuration of $\sigma, \tau$ and $\varsigma$. 


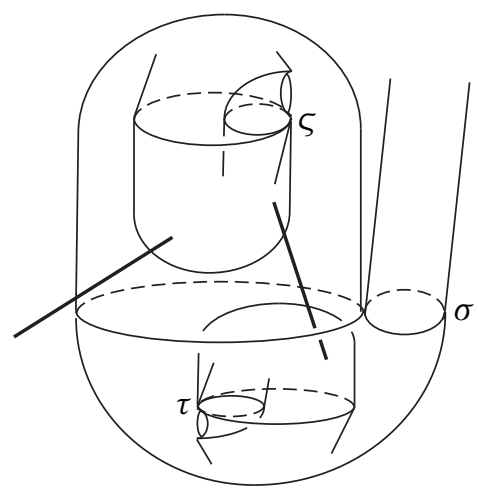

Figure 15

Case II.B.i Suppose $D_{\varsigma}$ has a maximum. Poke a neighborhood of $b$ in $D_{\varsigma}$ along $\gamma_{\sigma}$ away from $a$ and just past the maxima of $\gamma_{\sigma}$ as in the proof of Lemma 3.6. Since we have renamed so that $b$ is higher on $D_{\varsigma}$ than $b^{\prime}$ and $\gamma_{\sigma}$ is disjoint from $C$ above $b$, then this isotopy of $D_{\varsigma}$ creates a single inessential saddle. Use the isotopy in Lemma 3.3 to eliminate the resulting inessential saddle. The net result of these isotopies is to leave the number of saddles in $F_{C}$ and the number of maxima of $\left.h\right|_{K}$ unchanged. Additionally, this isotopy results in $a$ being the only point of intersection of $C$ with $\gamma_{\sigma}$. By Lemma 3.6, $C$ is not taut.

Case II.B.ii Suppose $D_{\varsigma}$ has a minimum. Let $S_{\varsigma}$ be the level surface containing $\varsigma$. $D_{\varsigma}$ divides the 3-ball below $S_{\varsigma}$ into two three balls $B_{\varsigma}$ and $B_{\varsigma}^{\prime}$ where we choose the labels in the unconventional way where $B_{\varsigma}$ contains $\sigma$ and may or may not contain $s_{2}^{5}$ in its boundary.

Case II.B.ii.a If $\left.h\right|_{K \cap B_{\varsigma}^{\prime}}$ has a maximum at $b^{\prime}$, then there must be a minimum of $\left.h\right|_{K}$ in $B_{\varsigma}^{\prime}$ or $\gamma_{\sigma}=\gamma_{\varsigma}$. However, the second possibility would imply $\left|\gamma_{\sigma} \cap C\right|>2$. So, we can assume there is a minimum of $\left.h\right|_{K}$ in $B_{\varsigma}^{\prime}$. By Lemma 3.2, we can assume $B_{\varsigma}^{\prime}$ does not contain $-\infty$ and $B_{\sigma}^{\prime}$ does not contain $+\infty$. As in Lemma 4.5, we can horizontally shrink and vertically lift the portion of $B_{\varsigma}^{\prime}$ lying below $b^{\prime}$ so that the resulting minimum of $D_{\varsigma}$ lies just below $b^{\prime}$ and horizontally shrink and vertically lower $B_{\sigma}^{\prime}$ so that it lies just above $\sigma$. By Lemma 4.3, $B_{\sigma}^{\prime}$ contains a maximum of $\left.h\right|_{K}$ and, by the argument above, $B_{\varsigma}^{\prime}$ contains a minimum of $\left.h\right|_{K}$. Since we have isotoped a minimum of $\left.h\right|_{K}$ above a maximum of $\left.h\right|_{K}$, bridge position for $K$ is not thin position.

Case II.B.ii.b Suppose $\left.h\right|_{K \cap B_{\varsigma}^{\prime}}$ has a minimum at $b^{\prime}$.

Suppose $c$ is above $b^{\prime}$ on $\gamma_{\tau}$. Since we have assumed $h(b)<h\left(b^{\prime}\right)$, then $h(a)<h(b)<$ $h\left(b^{\prime}\right)<h(c)$. Since $c$ is the highest point of $C \cap K$, then, if $D_{\tau}$ has a maximum, $C$ is 
not taut, by Lemma 3.6. If $D_{\tau}$ has a minimum, then $h(a)<h(c)$ implies $h(\sigma)<h(\varsigma)$ and we can appeal to the proof of Lemma 4.5 to show bridge position for $K$ is not thin position. Hence, we can assume $h(c)<h\left(b^{\prime}\right)$.

If $\varsigma \subset \partial\left(B_{\varsigma}\right)$, then $\varsigma$ is a removable saddle and $C$ is not taut, by Lemma 3.5. So, we can assume $\varsigma \subset \partial\left(B_{\varsigma}^{\prime}\right)$. Since $D_{\varsigma}$ contains two punctures, there can be at most three outermost disks in $F_{C}$ and, thus, at most one nonstandard saddle. With that in mind, at least one of $\sigma$ and $\tau$ is standard. Both $\sigma$ and $\tau$ are nonremovable, c-inessential saddles. We will assume $\sigma$ is standard (the case when $\tau$ is standard is proved analogously). This is the configuration depicted in Figure 15. Additionally, we can see $\left(B_{\sigma} \cup A_{\sigma}\right)^{c}$ is disjoint from $C \cap K$.

Since $B_{\sigma} \cup A_{\sigma}$ contains all of $C \cap K$, then, by Lemma 4.6, $\left(B_{\sigma} \cup A_{\sigma}\right)^{c}$ contains the unique nonstandard saddle for $F_{C}$. Since $\varsigma \subset \partial\left(B_{\varsigma}^{\prime}\right)$, the potentially nonstandard labeling of $B_{\varsigma}$ and $B_{\varsigma}^{\prime}$ we have assumed for this case matches the standard labeling as outlined in Section 2. With that in mind, $\sigma \in B_{\varsigma}$ and $\varsigma$ standard imply that $\left(B_{\sigma} \cup A_{\sigma}\right)^{c}$ and $\left(B_{\zeta} \cup A_{\zeta}\right)^{c}$ are disjoint. Since $\left(B_{\sigma} \cup A_{\sigma}\right)^{c}$ contains the unique nonstandard saddle for $F_{C}$, then $\left(B_{\varsigma} \cup A_{\varsigma}\right)^{c}$ does not contain a nonstandard saddle. Since $B_{\varsigma}$ meets $C$, $\left(B_{\varsigma} \cup A_{\varsigma}\right)^{c}$ is disjoint from $C \cap K$, and $\left(B_{\zeta} \cup A_{\zeta}\right)^{c}$ does not contain a nonstandard saddle, then $C$ is not taut, by Lemma 4.6.

Suppose $\left|C \cap \gamma_{\sigma}\right|=3$. Let $\sigma$ and $\tau$ be c-inessential saddles where $D_{\sigma} \cap K=a$ and $D_{\tau} \cap K=b$. By Lemma 3.5 and Lemma 3.6, both $\sigma$ and $\tau$ are nonremovable saddles and both $\left|\gamma_{\sigma} \cap C\right|$ and $\left|\gamma_{\tau} \cap C\right|$ are greater than one or else $C$ is not taut. If $b$ is not contained in $\gamma_{\sigma}$, then $\left|\gamma_{\tau} \cap C\right|=1$ and $C$ is not taut, by Lemma 3.6. So, we can assume $\gamma_{\sigma}=\gamma_{\tau}$. If $D_{\tau}$ has a minimum and $h(a)<h(b)$, then $\tau$ and $\sigma$ are pods. Hence, $C$ is not taut or bridge position for $K$ is not thin position for $K$, by Lemma 4.5. If $D_{\tau}$ has a minimum and $h(a)>h(b)$, then $\gamma_{\sigma}$ is disjoint from $C$ either above $a$ or below $b$. In either case, we can use the isotopy in Lemma 3.6 to eliminate one of $\sigma$ and $\tau$ and conclude $C$ is not taut. Hence, we can assume $D_{\tau}$ has a maximum and $b$ is contained in $\gamma_{\sigma}$. Since both $D_{\sigma}$ and $D_{\tau}$ contain maxima, then, up to relabeling of $\sigma$ and $\tau$, we can assume $h(b)>h(a)$. By Lemma 5.5, $C$ is not taut or bridge position for $K$ is not thin position.

Suppose $\left|C \cap \gamma_{\sigma}\right|=4$. Without loss of generality, we will assume that $D_{\sigma}$ has a maximum. Label the points of $C \cap \gamma_{\sigma}=\{a, b, c, d\}$ in order of increasing hight so that $h(a)<h(b)<h(c)<h(d)$.

If there are three or more c-inessential saddles in $F_{C}$, any possible arrangement satisfies the hypothesis of Lemma 5.5, showing $C$ is not taut or thin position for $K$ is not bridge position. Hence, we can assume there are at most two c-inessential saddles in $F_{C}$. 
Let $p_{\sigma}=D_{\sigma} \cap K$ and $p_{\tau}=D_{\tau} \cap K$. Since there are at most two c-inessential saddles and at least one nonstandard saddle in $F_{C}$, the points in $C \cap K$ which are not $p_{\sigma}$ and $p_{\tau}$ are the punctures of a 2-punctured outermost disk in $F_{C}$. By parity, these two punctures must be consecutive. Recall we have assumed $D_{\sigma}$ contains a maximum.

If $p_{\sigma}=d$ then, by Lemma 3.6, $C$ is not taut with respect to $\beta(K)$.

If $p_{\sigma}=c$, then $a$ and $b$ are the punctures on the twice punctured outermost disk and $p_{\tau}=d$. Since $p_{\tau}$ is above $p_{\sigma}$ on $\gamma_{\sigma}, C$ is not taut or bridge position for $K$ is not thin position, by Lemma 5.5.

If $p_{\sigma}=b$, then $c$ and $d$ are the punctures on the twice punctured outermost disk and $p_{\tau}=a$. Since $C$ is disjoint from $\gamma_{\sigma}$ below $a$, then, by Lemma 3.6, $D_{\tau}$ has a maximum or $C$ is not taut or bridge position for $K$ is not thin position. However, if $D_{\tau}$ has a maximum, then $p_{\sigma}$ is above $p_{\tau}$ on $\gamma_{\sigma}$ and $C$ is not taut or bridge position for $K$ is not thin position for $K$, by Lemma 5.5.

If $p_{\sigma}=a$, then $p_{\tau}$ is above $p_{\sigma}$ on $\gamma_{\sigma}$ and, by Lemma 5.5, $C$ is not taut or bridge position for $K$ is not thin position.

Theorem 5.7 Let $C$ be a c-incompressible Conway sphere in $S^{3}-K$. If $F_{C}$ contains a c-inessential saddle $\sigma$ and contains only standard saddles, then $C$ is not taut or bridge position for $K$ is not thin position.

Proof If $\sigma$ is removable then $C$ is not taut, by Lemma 3.5. Hence, we can assume $\sigma$ is nonremovable. Without loss of generality, we will assume $D_{\sigma}$ contains a maximum. By Lemma 5.1, $F_{C}$ contains only standard saddles implies $C$ is not taut or $F_{C}$ contains exactly two outermost disks $D_{\sigma}$ and $D_{\varsigma}$. Hence, we will assume $F_{C}$ contains exactly two outermost disks $D_{\sigma}$ and $D_{\varsigma}$. If $\sigma=\varsigma$, then there is a unique saddle in $F_{\sigma}$. Since $C$ is disjoint from the interior of $B_{\sigma}, \gamma_{\sigma}$ is disjoint from $C$ above $D_{\sigma} \cap K$. Thus, $C$ is not taut, by Lemma 3.6. Henceforth, we will assume that $\sigma$ is distinct from $\varsigma$. Let $a=D_{\sigma} \cap K$. We will proceed by cases.

Case I Suppose $C$ is disjoint from $\gamma_{\sigma}$ above $a$. By Lemma 3.6, $C$ is not taut.

Case II Suppose $C$ meets $\gamma_{\sigma}$ above $a$ in exactly one point $b$.

Case II.A If $b$ is not contained in $D_{\zeta}$, then poke a neighborhood of $b$ in $C$ along $\gamma_{\sigma}$ toward and just past the maximum of $\gamma_{\sigma}$ as in the proof of Lemma 3.6. Since $\gamma_{\sigma}$ is disjoint from $C$ above $b$, this isotopy leaves $K$ fixed while adding exactly one c-inessential saddle $\tau$ to $F_{C}$. If $b$ was not originally contained on a monotone disk of $F_{C}$, then $\tau$ is nonstandard. If $b$ was originally contained on a monotone disk of $F_{C}$ such that this disk has a unique maximum, then $\tau$ is nonstandard. If $b$ was originally 
contained on a monotone disk $D$ of $F_{C}$ such that this disk has a unique minimum, then $\partial(D)=\delta$ where $\delta$ is a saddle in $F_{C}$. In this case, $\delta$ is nonstandard after the isotopy. Hence, we can assume that, after the isotopy, $F_{C}$ contains a nonstandard saddle. Additionally, after this isotopy, $\gamma_{\sigma}$ is disjoint from $C$ above $a$. Hence, we can use the isotopy in the proof of Lemma 3.6 to eliminate $\sigma$. The net change to the number of saddles in $F_{C}$ under these two isotopies is zero. However, $F_{C}$ now contains both a c-inessential saddle and a nonstandard saddle. Thus, $C$ is not taut or bridge position for $K$ is not thin position, by Theorem 5.6. See Figure 16.
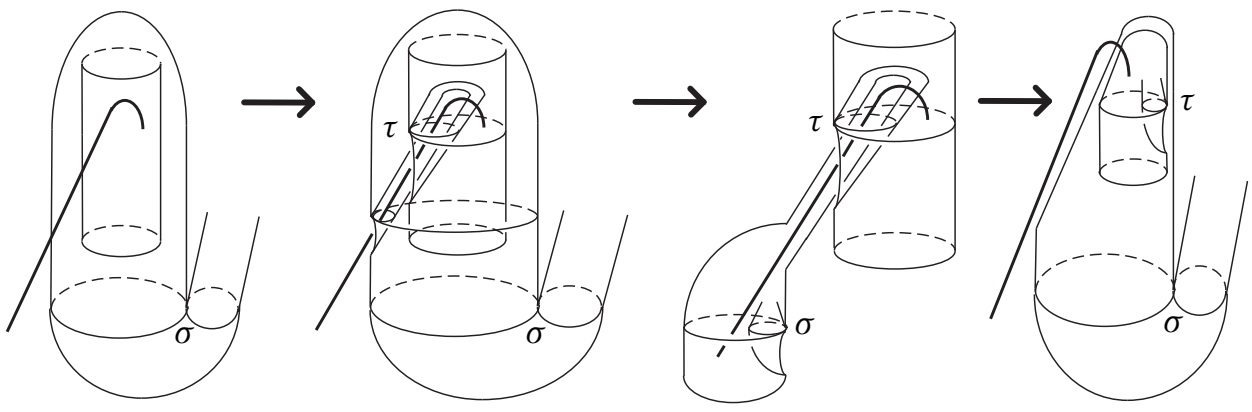

Figure 16

Case II.B.i If $b$ is contained in $D_{\varsigma}$ and $K \cap D_{\varsigma}=\{b\}$, then $C$ is not taut or bridge position for $K$ is not thin position for $K$, by Lemma 5.5 .

Case II.B.ii $b$ is contained in $D_{\varsigma}$ and $K \cap D_{\varsigma}=\left\{b, b^{\prime}\right\}$.

Case II.B.ii.a Suppose $D_{\varsigma}$ has a maximum and $h(b)>h\left(b^{\prime}\right)$. Poke a neighborhood of $b$ in $C$ along $\gamma_{\sigma}$ toward and just past the maximum of $\gamma_{\sigma}$ as in Lemma 3.6. Since $\gamma_{\sigma}$ is disjoint from $C$ above $b$, this isotopy leaves $K$ fixed while adding exactly one saddle $\tau$ to $F_{C}$. Since $D_{\varsigma}$ is disjoint from $K$ above $b, \tau$ is an inessential saddle. We can eliminate $\tau$ using the isotopy from Lemma 3.3. After these two isotopies, the number of saddles in $F_{C}$ has not been changed and $\gamma_{\sigma}$ is disjoint from $C$ above $a$. By Lemma 3.6, $C$ is not taut.

Case II.B.ii.b Suppose $D_{\varsigma}$ has a maximum and $h\left(b^{\prime}\right)>h(b)$. Poke a neighborhood of $b$ in $C$ along $\gamma_{\sigma}$ toward and just past the maximum of $\gamma_{\sigma}$ as in Lemma 3.6. Since $\gamma_{\sigma}$ is disjoint from $C$ above $b$, this isotopy leaves $K$ fixed while adding exactly one saddle $\tau$ to $F_{C}$. Since $D_{\varsigma}$ meets $K$ only in $b^{\prime}$ above $b$, then $\tau$ is a doubly c-inessential saddle. After this isotopy, $\gamma_{\sigma}$ is disjoint from $C$ above $a$. Hence, we can eliminate $\sigma$ by using the isotopy from Lemma 3.6. The net effect of these isotopies is to leave the number of saddles in $F_{C}$ unchanged and introduce a doubly c-inessential saddle $\tau$. By Lemma 5.3, $C$ is not taut or bridge position for $K$ is not thin position for $K$. 
Case II.B.ii.c Suppose $D_{\varsigma}$ has a minimum. Let $\varsigma=s_{1}^{\varsigma} \vee s_{2}^{\varsigma}$. Since $D_{\varsigma}$ is an outermost disk, then, up to relabeling, we can assume that $\partial\left(D_{\varsigma}\right)=s_{1}^{\varsigma}$. Let $S_{\varsigma}$ be the level surface containing $\varsigma . D_{\varsigma}$ cuts the three ball below $S_{\varsigma}$ in to two 3-balls $B_{\varsigma}$ and $B_{\varsigma}^{\prime}$ as described in Section 2. By hypothesis, $\varsigma$ is a standard saddle and, thus, is the boundary of a monotone disk $E_{\zeta}$. Since $C$ is embedded, $E_{\zeta}$ is contained completely in $B_{\sigma}$. If $E_{\zeta} \cap K \neq \varnothing$, then all four points of $C \cap K$ are contained in $B_{\sigma} \cup A_{\sigma}$ and $K$ is disjoint from $E_{\sigma}$. By Lemma 4.6, $C$ is not taut or bridge position for $K$ is not thin position for $K$. Hence we can assume that $E_{\zeta} \cap K=\varnothing$. Since all saddles in $F_{C}$ are standard, then, by Lemma 4.6, we can assume $\left(B_{\varsigma} \cup A_{\varsigma}\right)^{c}$ contains at least one point of $C \cap K$. If $\sigma$ is contained in $B_{\zeta}$, then $\left(B_{\zeta} \cup A_{\zeta}\right)^{c}$ is contained in $\operatorname{int}\left(A_{\sigma} \cup B_{\sigma}\right)$ and $A_{\sigma} \cup B_{\sigma}$ contains all four points of $C \cap K$. Then, by Lemma 4.6, $C$ is not taut or bridge position for $K$ is not thin position. Hence, we can assume that $\sigma$ is contained in $B_{\varsigma}^{\prime}$. Since $\sigma$ is contained in $B_{\varsigma}^{\prime}$, then $h_{K \cap B_{\varsigma}}$ has a minimum at $b$. If $b^{\prime}$ is a minimum of $h_{K \cap B_{\varsigma}}$, then $\varsigma$ is a removable saddle and $C$ is not taut, by Lemma 3.5. Hence, we can assume that $b^{\prime}$ is a maximum of $h_{K} \cap B_{\varsigma}$. This leads us to the following two possibilities. After these observations, one possible arrangement of $K, \sigma$ and $\varsigma$ is depicted in Figure 17.

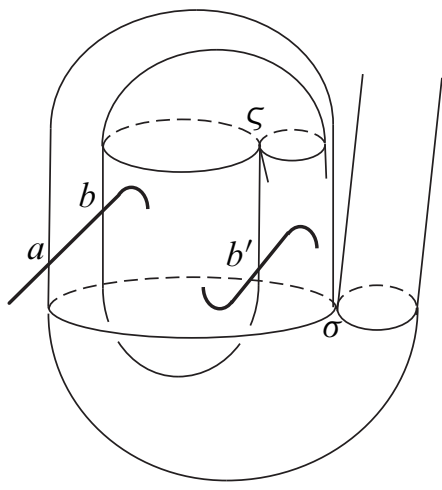

Figure 17

Case II.B.ii.c.1 $D_{\varsigma}$ has a minimum, $B_{\varsigma}^{\prime}$ contains $\sigma, b^{\prime}$ is a maximum of $h_{K} \cap B_{\varsigma}$ and $h(b)<h\left(b^{\prime}\right)$. Since $b^{\prime}$ is a maximum of $\left.h\right|_{K \cap B_{\varsigma}}$ and $b^{\prime}$ is not on $\gamma_{\sigma}$, then there is a minimum of $\left.h\right|_{K}$ in $B_{\varsigma}$.

By appealing to Lemma 3.2, we can assume $B_{\sigma}^{\prime}$ does not contain $+\infty$ and $B_{\varsigma}$ does not contain $-\infty$. Since $\sigma$ is a nonremovable, c-inessential saddle in $F_{C}$ such that $D_{\sigma}$ contains a maximum, then $h_{K \cap B_{\sigma}^{\prime}}$ has a local maximum at $K \cap D_{\sigma}$. Since $\left.h\right|_{K \cap B_{\sigma}^{\prime}}$ has a local maximum at $a$, we can appeal to the isotopy in Lemma 3.5 to horizontally shrink and vertically lower $B_{\sigma}^{\prime}$ until $h\left(B_{\sigma}^{\prime}\right)$ lies between $h(\sigma)$ and $h(\sigma)+\varepsilon$ for any 
$\varepsilon>0$. Since $b$ is the lowest point of intersection of $K$ with $D_{\varsigma}$ we can horizontally shrink and vertically raise the portion of $B_{\varsigma}$ that lies below $b$ until $h\left(B_{\varsigma}\right)$ is contained between $h(b)-\varepsilon$ and $h(\varsigma)$ for any $\varepsilon>0$. Since $h(b)>h(a)$, then $h(b)>h(\sigma)$. Hence, we can lower $B_{\sigma}^{\prime}$ and raise $B_{\varsigma}$ until $B_{\sigma}^{\prime}$ lies strictly below $B_{\varsigma}$ without changing the number of maxima of $h_{K}$ and without introducing any new saddles. However, by Lemma $4.3, B_{\sigma}^{\prime}$ contains a maximum of $\left.h\right|_{K}$ and, by the above argument, $B_{\varsigma}$ contains a minimum of $\left.h\right|_{K}$. Thus, bridge position for $K$ is not thin position.

Case II.B.ii.c.2 $D_{\varsigma}$ has a minimum, $B_{\varsigma}^{\prime}$ contains $\sigma, b^{\prime}$ is a maximum of $h_{K \cap B_{\varsigma}}$ and $h\left(b^{\prime}\right)<h(b)$. This is the situation depicted in Figure 17. Let $\gamma_{b^{\prime}}$ be the maximal monotone subarc of $K$ that contains $b^{\prime}$. If $\gamma_{b^{\prime}}$ meets $C$ at any point other than $b^{\prime}$ then $B_{\sigma} \cup A_{\sigma}$ contains all four points of $C \cap K$ and, by Lemma 4.6, $C$ is not taut or bridge position of $K$ is not thin position. Hence, we can assume that $\gamma_{b^{\prime}} \cap C=\left\{b^{\prime}\right\}$.

Poke a neighborhood of $b^{\prime}$ in $C$ along $\gamma_{b^{\prime}}$ toward and just past the minimum of $\gamma_{b^{\prime}}$ as in Lemma 3.6. Since $\gamma_{b^{\prime}}$ is disjoint from $C$ below $b^{\prime}$, this isotopy leaves $K$ fixed while adding exactly one saddle $\tau$ to $F_{C}$. Since $D_{\varsigma}$ is disjoint from $K$ below $b^{\prime}$, then $\tau$ is an inessential saddle. We can eliminate $\tau$ using the isotopy from Lemma 3.3. After these two isotopies, the number of saddles in $F_{C}$ has not been changed and both points of intersection of $K$ with $D_{\varsigma}$ are minima of $\left.h\right|_{K \cap B_{\varsigma}}$. Hence, $\varsigma$ is a removable saddle and $C$ is not taut, by Lemma 3.5. See Figure 18.

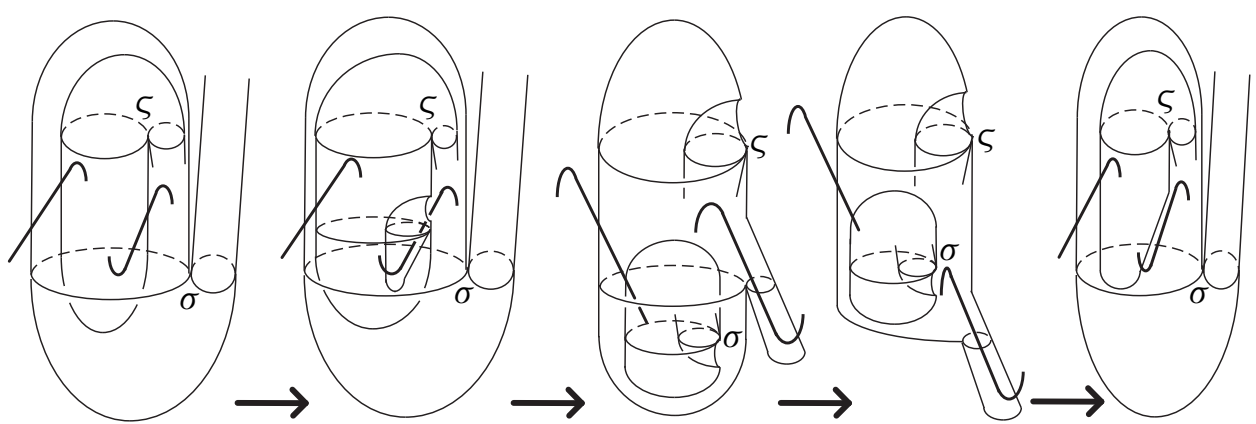

Figure 18

Case II.B.iii Assume $b$ is contained in $D_{\varsigma}$ and $K \cap D_{\varsigma}=\left\{b, b^{\prime}, b^{\prime \prime}\right\}$. In this case, all four points of $C \cap K$ are contained in $B_{\sigma} \cup A_{\sigma}$ and $\sigma$ is a standard saddle such that $K$ is disjoint from $E_{\sigma}$. Since $\left(B_{\sigma} \cup A_{\sigma}\right)^{c}$ contains only standard saddles and is disjoint from $K \cap C$, then $C$ is not taut, by Lemma 4.6.

Case III Suppose $C$ meets $\gamma_{\sigma}$ above $a$ in exactly two points $b$ and $c$ where $h(a)<$ $h(b)<h(c)$. 
If both $b$ and $c$ are disjoint from $D_{\varsigma}$, then $\varsigma$ is a nonremovable c-inessential saddle or else $C$ is not taut. If $\gamma_{\varsigma}$ is distinct from $\gamma_{\sigma}$, then $\gamma_{\varsigma}$ consists of a single point and $C$ is not taut, by Lemma 3.6. If $\gamma_{\varsigma}=\gamma_{\sigma}$, then $D_{\varsigma} \cap K$ is below $a$ on $\gamma_{\sigma}$. If $D_{\varsigma}$ has a maximum, then $C$ is not taut or bridge position for $K$ is not thin position, by Lemma 5.5. If $D_{\varsigma}$ has a minimum, then $C$ is not taut, by Lemma 3.6. Hence, we can assume that $b$ or $c$ lie on $D_{\varsigma}$.

If $D_{\varsigma}$ meets $K$ in a single point, then that point is either $b$ or $c$ and $\varsigma$ is a c-inessential saddle. By Lemma 5.5, $C$ is not taut or bridge position for $K$ is not thin position. If $D_{\varsigma}$ meets $K$ in two points, one of which is not contained in the set $\{b, c\}$, then $B_{\sigma} \cup A_{\sigma}$ contains all four points of $C \cap K$ and, by Lemma 4.6, $C$ is not taut or bridge position for $K$ is not thin position. If $D_{\varsigma}$ meets $K$ in three points, then $B_{\sigma} \cup A_{\sigma}$ contains all four points of $C \cap K$ and, by Lemma 4.6, $C$ is not taut or bridge position for $K$ is not thin position. Hence, we can assume $D_{\varsigma}$ meets $K$ in exactly the two points $b$ and $c$.

Case III.A Suppose $D_{\varsigma}$ has a maximum and $D_{\varsigma}$ meets $K$ in exactly the two points $b$ and $c$. Poke a neighborhood of $c$ in $C$ along $\gamma_{\sigma}$ toward and just past the maximum of $\gamma_{\sigma}$ as in Lemma 3.6. Since $\gamma_{\sigma}$ is disjoint from $C$ above $c$, this isotopy leaves $K$ fixed while adding exactly one saddle $\tau$ to $F_{C}$. Since $D_{\zeta}$ is disjoint from $K$ above $c$, then $\tau$ is an inessential saddle. We can eliminate $\tau$ using the isotopy from Lemma 3.3. This is illustrated in Figure 19. After these two isotopies, neither the number of critical points of $\left.h\right|_{K}$ nor the number of saddles in $F_{C}$ has been changed and $C$ meets $\gamma_{\sigma}$ above $a$ in exactly one point. Hence, we have reduced this case to Case II and can conclude $C$ is not taut or bridge position for $K$ is not thin position.

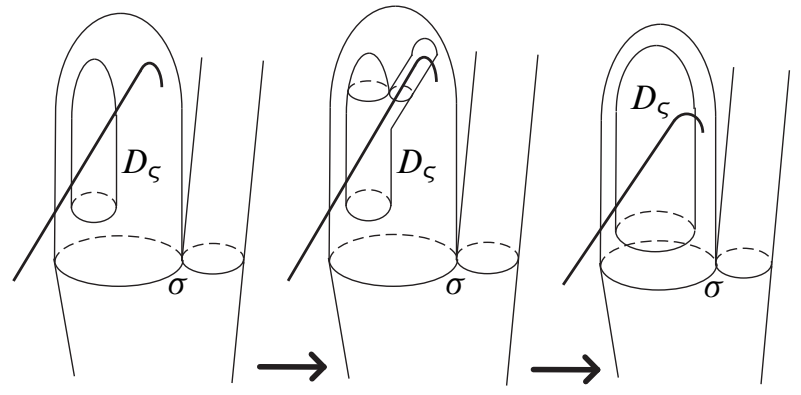

Figure 19

Case III.B Suppose $D_{\varsigma}$ has a minimum and $D_{\varsigma}$ meets $K$ in exactly the two points $b$ and $c$. Let $S_{c}$ be a level surface just above $c$. The portion of $D_{\varsigma}$ that lies below $S_{c}$ cuts the 3-ball below $S_{c}$ into two 3-balls $B_{c}$ and $B_{c}^{\prime}$ where we choose the labeling 
such that $B_{c}^{\prime}$ contains $\sigma$. The curve $D_{\varsigma} \cap S_{c}$ cuts $S_{c}$ into two disks $D_{c}$ and $D_{c}^{\prime}$ where $D_{c}$ meets $B_{c}$. If $D_{c}$ is disjoint from $K$, then we can use an innermost disk argument and the fact that $C$ is incompressible to isotope $C$ to be disjoint from $D_{c}$ while leaving $K$ fixed and possibly decreasing the number for saddles of $F_{C}$. After this isotopy, $D_{c}$ is a compressing disk for $C$, contradicting the fact that $C$ is incompressible. Hence, we can assume that $K$ is not disjoint from $D_{c}$. Since $K$ is not disjoint from $D_{c}$ and $b$ is connected to $c$ in $B_{c}$ via a monotone subarc of $K$, then there must be a minimum of $\left.h\right|_{K}$ in $B_{c}$. By appealing to Lemma 3.2, we can assume $B_{\sigma}^{\prime}$ does not contain $+\infty$ and $B_{c}$ does not contain $-\infty$. Since $\sigma$ is a nonremovable, c-inessential saddle in $F_{C}$ such that $D_{\sigma}$ contains a maximum, then $h_{K \cap B_{\sigma}^{\prime}}$ has a local maximum at $K \cap D_{\sigma}$. Since $\left.h\right|_{K \cap B_{\sigma}^{\prime}}$ has a local maximum at $a$, we can appeal to the isotopy in Lemma 3.5 to horizontally shrink and vertically lower $B_{\sigma}^{\prime}$ until $h\left(B_{\sigma}^{\prime}\right)$ lies between $h(\sigma)$ and $h(\sigma)+\varepsilon$ for any $\varepsilon>0$. Since $D_{c}$ is disjoint from $K$ below $b$, we can horizontally shrink and vertically rase the portion of $B_{c}$ that lies below $b$ until $h\left(B_{c}\right)$ is contained between $h(b)-\varepsilon$ and $h\left(S_{c}\right)$ for any $\varepsilon>0$. Since $h(b)>h(a)$, then $h(b)>h(\sigma)$. Hence, we can lower $B_{\sigma}^{\prime}$ and raise $B_{c}$ until $B_{\sigma}^{\prime}$ lies strictly below $B_{c}$ without changing the number of maxima of $h_{K}$ and without introducing any new saddles. However, by Lemma 4.3, $C$ is not taut or $B_{\sigma}^{\prime}$ contains a maximum of $\left.h\right|_{K}$ and, by the above argument, $B_{c}$ contains a minimum of $\left.h\right|_{K}$. Thus, $C$ is not taut or bridge position for $K$ is not thin position for $K$.

Case IV Suppose $C$ meets $\gamma_{\sigma}$ above $a$ in exactly three points. In this case, all four points of $C \cap K$ are contained in $B_{\sigma} \cup A_{\sigma}$ and $\sigma$ is a standard saddle such that $K$ is disjoint from $E_{\sigma}$. Since $\left(B_{\sigma} \cup A_{\sigma}\right)^{c}$ contains only standard saddles and is disjoint from $K \cap C$, then $C$ is not taut, by Lemma 4.6.

Theorem 5.8 Let $C$ be a c-incompressible Conway sphere in $S^{3}-K$. If $C$ is taut and $F_{C}$ contains a c-inessential saddle, then bridge position for $K$ is not thin position for $K$.

Proof If $F_{C}$ contains a nonstandard saddle, we are done by Theorem 5.6. If $F_{C}$ does not contain a nonstandard saddle, we are done by Theorem 5.7.

Recall the definition of nested from Section 3.

Definition 5.9 A Conway sphere is worm-like if, for every saddle $\sigma=s_{1}^{\sigma} \vee s_{2}^{\sigma}$, each of $s_{1}^{\sigma}$ and $s_{2}^{\sigma}$ cut $C$ into two twice punctured disks and every saddle in $F_{C}$ is nested with respect to the same side of $C$.

Theorem A If $C$ is a taut c-incompressible Conway sphere in $S^{3}-K$ and bridge position for $K$ is thin position, then $C$ is worm-like. 
Proof By Theorem 5.8, $F_{C}$ contains no c-inessential saddles. By the remark following Lemma 5.1, $F_{C}$ contains no nonstandard saddles. Thus, there are exactly two outermost disks in $F_{C}$ each meeting $K$ exactly twice. Since every saddle in $F_{C}$ is standard, then every saddle $\sigma$ bounds a monotone disk $E_{\sigma}$. If any $E_{\sigma}$ meets $K$, then one of the two outermost disks in $F_{C}$ meets $K$ in less than two points. Hence, every $E_{\sigma}$ is disjoint from $K$. If any $s_{i}^{\sigma}$ bounds a disk $D$ in $C$ that is disjoint from $K$ or meets $K$ once, then an outermost saddle in $F_{D}$ is an inessential or c-inessential saddle. Hence, every $s_{i}^{\sigma}$ cuts $C$ into two twice punctured disks. Let $C$ decompose $S^{3}$ into two 3-balls $B_{1}$ and $B_{2}$. If $F_{C}$ contains saddles nested with respect to $B_{1}$ and saddles nested with respect to $B_{2}$, then there are adjacent saddles in $F_{C}$ nested with respect to distinct 3-balls. However, this contradicts $C$ being taut, by Lemma 3.7. Thus, $C$ is worm-like.

\section{Conway products and bridge inequalities}

Let $K_{1} \subset S_{1}^{3}$ and $K_{2} \subset S_{2}^{3}$ be links embedded in distinct 3-spheres. For each $i=1,2$ let $\tau_{i}$ be $\operatorname{arcs}$ in $S_{i}^{3}$ such that $\partial \tau_{i} \subset K_{i}$ but $\tau_{i}$ is otherwise disjoint from $K_{i}$. Let $\eta\left(\tau_{i}\right)$ be a regular closed neighborhood of $\tau_{i}$, then $\eta\left(\tau_{i}\right) \cap K_{i}$ is a trivial tangle and $\partial\left(\eta\left(\tau_{i}\right)\right)$ is a Conway sphere for $K_{i}$. Let $B_{i}=S_{i}^{3}-\operatorname{int}\left(\eta\left(\tau_{i}\right)\right)$.

Definition 6.1 Let $K_{1} *_{c} K_{2}$ (the generalized Conway product of $K_{1}$ and $K_{2}$ ) denote the link in $S^{3}$ formed by removing $\operatorname{int}\left(\eta\left(\tau_{i}\right)\right)$ from $S_{i}^{3}$ and gluing $\partial\left(B_{1}\right)$ to $\partial\left(B_{2}\right)$ via a homeomorphism which sends $K_{1} \cap \partial\left(B_{1}\right)$ to $K_{2} \cap \partial\left(B_{2}\right)$.

The image $C$ of $\partial\left(\eta\left(\tau_{1}\right)\right)$ and $\partial\left(\eta\left(\tau_{2}\right)\right)$ after their identification, is the Conway sphere of the generalized Conway product.

The isotopy class of $K_{1} *_{c} K_{2}$ is dependent on the isotopy classes of $K_{1}$ and $K_{2}$, the isotopy classes of $\tau_{1}$ and $\tau_{2}$, and the gluing homeomorphism. In fact, there are infinitely many distinct links $K_{1} *_{c} K_{2}$ for any pair of links $K_{1}$ and $K_{2}$. An example of a generalized Conway product is given in Figure 25.

In this section, we will use Theorem A to relate the bridge number of the factor links $K_{1}$ and $K_{2}$ to the bridge number of their generalized Conway product $K_{1} *_{c} K_{2}$. To do so, we restrict only to generalized Conway products were $C$ is c-incompressible and bridge position for $K_{1} *_{c} K_{2}$ is thin position. In addition, if $C$ is taut, then, by Theorem A, $C$ is worm-like. In particular, all saddles of $F_{C}$ are nested with respect to $B_{2}$, up to labeling. With this labeling, we say $K_{1}$ is the distinguished factor of $K_{1} *_{c} K_{2}$. 
Let $K$ be a link in $S^{3}$ and $C$ be a c-incompressible Conway sphere. If $C$ is taut and bridge position for $K$ is thin position, then, by Theorem A, there are exactly two outermost disks in $F_{C}, D_{1}$ and $D_{2}$, and each $D_{i}$ meets $K$ exactly twice. We use the following labeling convention: $\left\{x_{1}^{i}, x_{2}^{i}\right\}=K \cap D_{i}$ and $h\left(x_{1}^{i}\right)>h\left(x_{2}^{i}\right)$ for $i=1,2$. We will want to keep track of the following properties:

(1) Is $x_{j}^{i}$ a local minimum or maximum of $\left.h\right|_{K \cap B_{1}}$ for $i=1,2$ and $j=1,2$ ?

(2) Does $\left.h\right|_{D_{i}}$ have a unique local minimum or maximum for $i=1,2$ ? (That is, is $D_{i}$ a cap or a cup?)

To accomplish this we define a 3-tuple labeling $(x, y, z) \epsilon\{m, M\}^{3}$ for each $D_{i}$ where $x=m$ (resp. $M$ ) if $x_{1}^{i}$ is a minimum (resp. maximum) of $\left.h\right|_{K \cap B_{1}}, y=m$ (resp. $M$ ) if $x_{2}^{i}$ is a minimum (resp. maximum) of $\left.h\right|_{K \cap B_{1}}$, and $z=m$ (resp. $M$ ) if $\left.h\right|_{D_{i}}$ has a unique minimum (resp. maximum).

As an example, the disk in Figure 20 is labeled $(M, m, m)$.

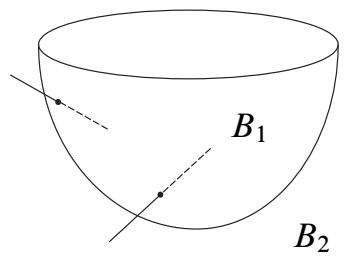

Figure 20

Lemma 6.2 If $K=K_{1} *_{c} K_{2}$ is a generalized Conway product such that $C$ is cincompressible and bridge position for $K$ is thin position, then there is an isotopy of $K$ and $C$ resulting in $\left.h\right|_{K}$ having $\beta(K)$ maxima, $C$ being taut, and $\left.h\right|_{K}$ having at least one maximum or minimum in $B_{2}$.

Proof By isotoping $C$ so that $F_{C}$ has the fewest number of saddles subject to $\left.h\right|_{K}$ having $\beta(K)$ maxima, we can assume $C$ is taut. By Theorem A, we can assume $C$ is worm-like. If $F_{C}$ contains saddles then $D_{1}$ and $D_{2}$ are defined as in the above discussion. If $F_{C}$ has no saddles, then let $s$ be a level curve in $F_{C}$ which separates two points in $C \cap K$ from two others. The two components of $C-s$ are the monotone, twice-punctured disks $D_{1}$ and $D_{2}$.

We will proceed by cases using the 3-tuple labeling of $D_{1}$ and $D_{2}$. An underscore in a coordinate of a labeling will indicate $m$ or $M$. (That is, $(m,-M)$ represents $(m, m, M)$ or $(m, M, M))$. 
Suppose $D_{i}$ is labeled $(M, M, M)$ or $(m, m, m)$ for $i \epsilon\{1,2\}$. Let $\sigma$ be the saddle in $F_{C}$ such that $\partial\left(D_{i}\right) \subset \sigma$. Since all saddles are nested with respect to $B_{1}$ a label of $(M, M, M)$ or $(m, m, m)$ implies $\sigma$ is removable and contradicts the assumption that $C$ is taut, by Lemma 3.5.

Suppose one of $D_{i}$ for $i=1,2$ is labeled $\left(m,{ }_{-}, M\right)$ or $\left({ }_{-}, M, m\right)$. Up to renaming of the disks and reflection in a level sphere, we can assume $D_{1}$ has the 3-tuple label $\left(m,{ }_{-}, M\right)$. Let $\sigma$ be the saddle in $F_{C}$ such that $\partial\left(D_{1}\right)=s_{1}^{\sigma}$. Let $L$ be the level surface containing $\sigma$. Let $\gamma$ be the maximal monotone strand of $K \cap B_{1}$ that contains $x_{1}^{1}$, so $\gamma$ ascends from $x_{1}^{1}$ into $B_{1}$.

Suppose $C$ meets $\gamma$ above $x_{1}$ in a point $a$. If $a$ is contained in $D_{2}$, then $D_{2}$ is entirely contained in $B_{\sigma} \cup A_{\sigma}$ and $B_{\sigma} \cup A_{\sigma}$ contains all four points of $C \cap K$. Since $F_{C}$ contains only standard saddles, then we contradict $C$ being taut or bridge position for $K$ being thin position, by Lemma 4.6. If $a$ is not contained in $D_{2}$, then $a$ must be $x_{2}^{1}$. However, this would place $x_{2}^{1}$ higher with respect to $h$ than $x_{1}^{1}$. This is a contradiction to how we defined $x_{1}^{1}$. Thus, we may assume that $\gamma$ is disjoint from $C$ above $x_{1}^{1}$.

Poke a small neighborhood of the point $x_{1}^{1}$ in $C$ along $\gamma$ toward and slightly past the maximum of $\gamma$ as in the proof of Lemma 3.6. This isotopy fixes $K$ and, since $\gamma$ is disjoint from $C$ above $x_{1}^{1}$, adds a single saddle $\tau$ to $F_{C}$. Since $K$ is disjoint from $D_{1}$ above $x_{1}^{1}$, then $\tau$ is an inessential saddle. Use the isotopy from Lemma 3.3 to remove $\tau$. This isotopy preserves the number of maxima of $\left.h\right|_{K}$ and results in $C$ being taut, but alters $D_{1}$ so that its new label is $\left(M,{ }_{-}, M\right)$. See Figure 5 .

Therefore, we can assume the labels of $D_{1}$ and $D_{2}$ are both chosen from the set $\{(M, m, m),(M, m, M)\}$. The disks corresponding to these two possible labelings are depicted in Figure 21.

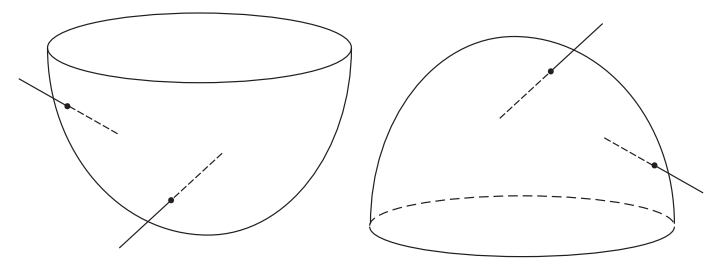

Figure 21

Suppose $D_{1}$ is labeled $(M, m, M)$ and $D_{2}$ is labeled $(M, m, m)$. Let $\alpha$ be the component of $K \cap B_{2}$ with an endpoint $x_{1}^{1}$. If $\alpha$ contains a maximum or minimum of $\left.h\right|_{K}$, then we are done. If not, then $\alpha$ is monotone and the other endpoint of $\alpha$ must be $x_{2}^{2}$. This leaves $x_{2}^{1}$ and $x_{1}^{2}$ connected by $\beta$, a component of $K \cap B_{2}$. The 
monotonicity of $\alpha$ ensures $h\left(x_{2}^{2}\right)>h\left(x_{1}^{1}\right)$. Since $h\left(x_{1}^{1}\right)>h\left(x_{2}^{1}\right), h\left(x_{1}^{2}\right)>h\left(x_{2}^{2}\right)$ and $h\left(x_{2}^{2}\right)>h\left(x_{1}^{1}\right)$, then $h\left(x_{2}^{1}\right)>h\left(x_{1}^{2}\right)$. However, $x_{2}^{1}$ is labeled $m$ and $x_{1}^{2}$ is labeled $M$, so there must be both a minimum and a maximum of $\left.h\right|_{K}$ in $\beta \subset B_{2}$. See Figure 22. Thus, $\left.h\right|_{K \cap B_{2}}$ contains at least one minimum or maximum. This result follows analogously for the other possible labelings of $D_{1}$ and $D_{2}$.

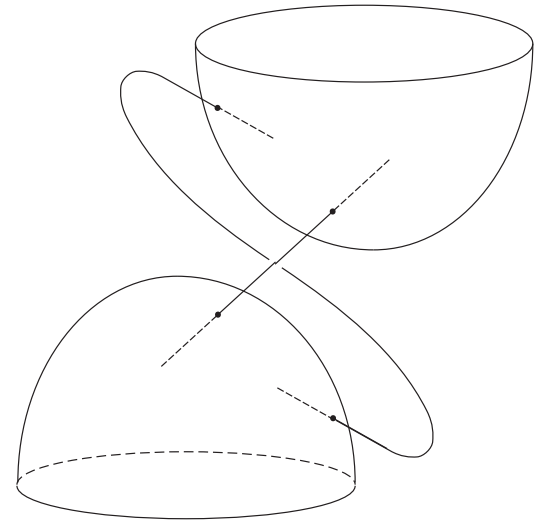

Figure 22

Theorem B If $K=K_{1} *_{c} K_{2}$ is a generalized Conway product such that $C$ is cincompressible and bridge position for $K$ is thin position, then $\beta(K) \geq \beta\left(K_{1}\right)-1$ where $K_{1}$ is the distinguished factor.

Proof Choose $C$ taut. By Theorem A and Lemma 6.2, we can assume $C$ is worm-like and $\left.h\right|_{K}$ has at least one maximum in $B_{2}$ (the case where $\left.h\right|_{K}$ has one minimum in $B_{2}$ is proved analogously). To prove the theorem, we need only prove that the number of maxima of $\left.h\right|_{K}$ in $B_{1}$ is greater than or equal to $\beta\left(K_{1}\right)-2$. The theorem will then follow since $\beta(K)=$ (number of maxima of $\left.h\right|_{K}$ in $\left.B_{1}\right)+$ (number of maxima of $\left.h\right|_{K}$ in $\left.B_{2}\right)$.

First, we analyze the case where $F_{C}$ contains no saddles. In this case, there is a level preserving isotopy of $S^{3}$ taking $C$ to a standard round 2-sphere. Such an isotopy preserves the number and nature of maxima of $\left.h\right|_{K}$ in $B_{1}$. As in Lemma 6.2, a point in $K \cap C$ is labeled with an $m$ if it is a local minimum of $\left.h\right|_{K \cap B_{1}}$ and is labeled with an $M$ if it is a local maximum of $\left.h\right|_{K \cap B_{1}}$. The link $K_{1}$ can be recovered from $K \cap B_{1}$ by gluing a rational tangle $T$ to $K \cap B_{1}$ along their common 4 -punctured sphere boundary. If more points of $K \cap C$ are labeled with an $M$, take $T$ to lie above $C$. If more are labeled with an $m$, take $T$ to lie below $C$. See Figure 23. Since the portion 
of the rational tangle lying in the region labeled $R$ can be taken to be monotone with respect to $h$, this rational completion causes the creation of at most two new maxima. The number of maxima of the resulting embedding of $K_{1}$ is at most two more than the number of maxima of $\left.h\right|_{K}$ in $B_{1}$. Hence, the number of maxima of $\left.h\right|_{K}$ in $B_{1}$ is greater than or equal to $\beta\left(K_{1}\right)-2$.

(Note: If $F_{C}$ has no saddles, we get the analogous estimate that the number of maxima of $\left.h\right|_{K}$ in $B_{2}$ is greater than or equal to $\beta\left(K_{2}\right)-2$. Hence, in this special case, we get the additional inequality $\beta(K) \geq \beta\left(K_{1}\right)+\beta\left(K_{2}\right)-4$.)

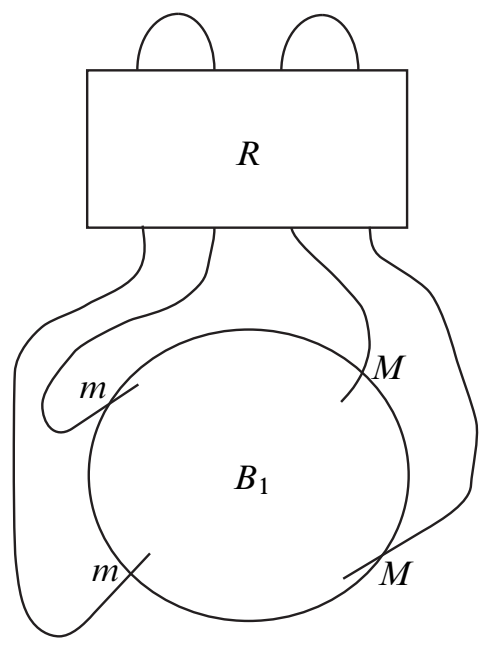

Figure 23

We now assume $F_{C}$ contains saddles. To establish the desired inequality in this general setting, we build an isotopy of $S^{3}$ which takes $B_{1}$ to a standard round 3-ball and preserves the number and nature of critical points of $\left.h\right|_{K}$ in $B_{1}$. This isotopy, however, does not preserve the number of critical points of $\left.h\right|_{K}$ in $B_{2}$. Let $D_{1}$ and $D_{2}$ be the two outermost disks for $F_{C}$. $F_{D_{1}}$ is a collection of circles and one point corresponding to a maximum of $\left.h\right|_{C}$ (if the point is a minimum, the case is analogous). Recall the terminology introduced in Section 2. Let $\sigma$ be the saddle in $F_{C}$ such that $D_{\sigma}=D_{1}$. By appealing to the proof of Lemma 3.2, we can assume $B_{\sigma}$ does not meet $+\infty$. Each point of $K \cap D_{1}\left(x_{1}^{1}\right.$ and $\left.x_{2}^{1}\right)$ receives a label of $M$ or $m$ as described above.

Since $\left.h\right|_{D_{1}}$ has a maximum as the unique critical point, we can horizontally shrink and vertically lower $B_{\sigma}$ until $D_{1}$ lies just above $D_{1}^{\sigma}$. Let $C^{*}$ be the image of $C$ and $D_{1}^{*}$ be the image of $D_{1}$ under this isotopy and let $p$ be the unique maximum of $\left.h\right|_{D_{1}^{*}}$. Let $J$ be the level surface containing $p$. Since we assume the $h$ restricts to a Morse 
function on $C^{*}, J \cap C^{*}$ consists of the point $p$ and a collection of circles. One such circle $c_{2}$ is parallel in $C^{*}$ to $s_{2}^{\sigma}$. By isotoping $D_{1}^{*}$ close enough to $D_{1}^{\sigma}$, we can choose a point $b$ in $c_{2}$ and an arc $\alpha$ in $J$ that is disjoint from $C^{*}$ except at its boundary, $\{b, p\}$. Choose another $\operatorname{arc} \beta$ in $C^{*}$ that does not meet $K$, has boundary $\{b, p\}$ and is transverse to $F_{C}$ everywhere except where it passes through $s_{1}^{\sigma} \cap s_{2}^{\sigma}$. Having made $D_{1}^{*}$ sufficiently close to $D_{1}^{\sigma}$, we can assume $\alpha$ and $\beta$ cobound a disk $F$ that is vertical with respect to $h$, disjoint from $K$, and disjoint from $C$ except along $\beta$. Isotope $C^{*}$ along $F$ to effectively cancel a saddle with a maximum. See Figure 24.

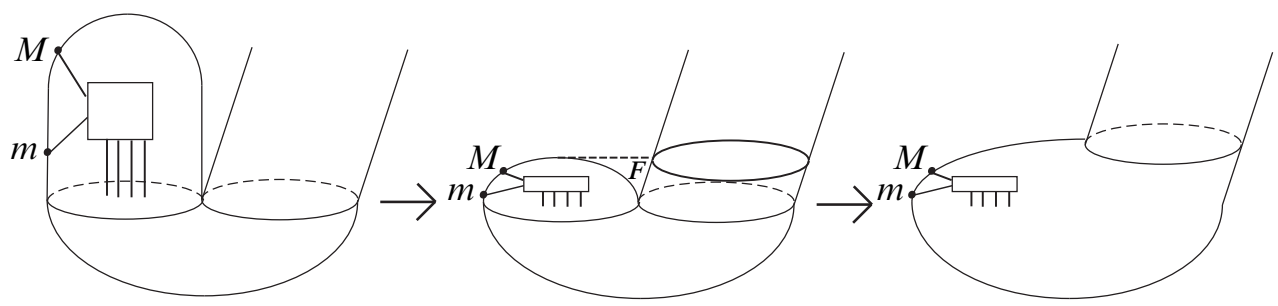

Figure 24

Repeat this process to produce an isotopy that preserves the number and nature of critical points of $\left.h\right|_{K \cap B_{1}}$ and takes $C$ to a standard round sphere. By the above argument, the number of maxima of $\left.h\right|_{K}$ in $B_{1}$ is greater than or equal to $\beta\left(K_{1}\right)-2$. This completes the proof of the theorem.

\section{An example}

In Figure 25, $K_{1}$ is the connect sum of four trefoils and $K_{2}$ is an index 2 satellite link of the trefoil. Schubert's seminal work on bridge number tells us that $\beta\left(K_{1}\right)=5$ and $\beta\left(K_{2}\right) \geq 4$. Since Figure 25 gives a presentation of $K_{2}$ with exactly 4 maxima, we conclude that $\beta\left(K_{2}\right)=4$. The link $K_{1} *_{c} K_{2}$ depicted in Figure 25 is an index 2 satellite link of the trefoil. Again Schubert's results tell us that $\beta\left(K_{1} *_{c} K_{2}\right) \geq 4$ and again we have a presentation of $K_{1} *_{c} K_{2}$ with exactly 4 maxima. Hence, $\beta\left(K_{1} *_{c} K_{2}\right)=4=\beta\left(K_{1}\right)-1$. By analyzing the Conway sphere of $K_{1} *_{c} K_{2}$ in the projection depicted in Figure 25, we see that $K_{1}$ is indeed the distinguished factor.

To prove the lower bound in Theorem B is tight it is left to show that $C$ in this example is c-incompressible and the projection of $K_{1} *_{c} K_{2}$ in Figure 25 is thin. It is easy to show $K_{1} *_{c} K_{2}$ in the example is prime and, thus, $C$ is c-incompressible. Bridge position for $K_{1} *_{c} K_{2}$ in this example is thin position. The proof uses standard ideas but has been omitted due to its length. Hence, the bound presented in Theorem B is tight. 


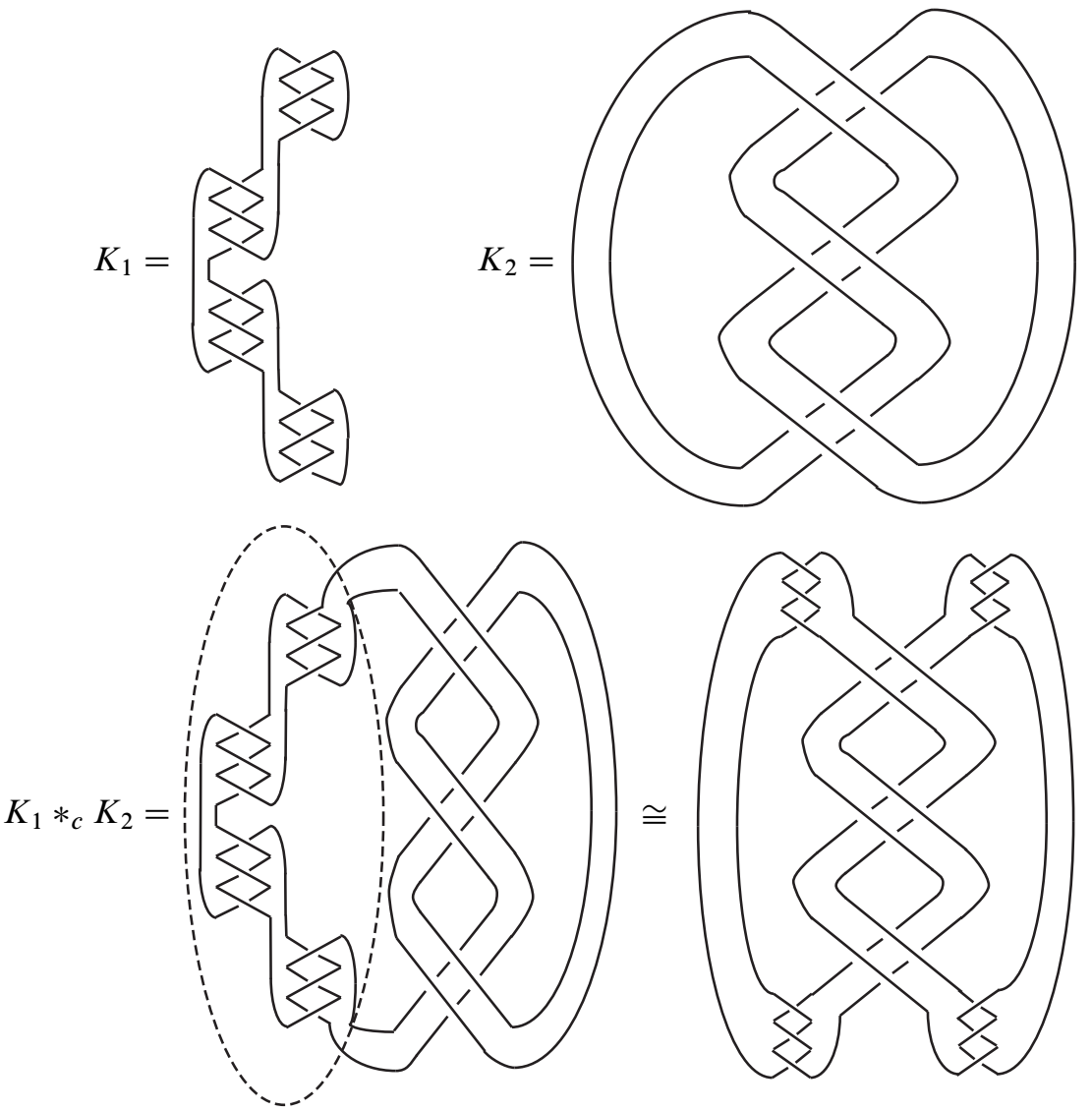

Figure 25

\section{References}

[1] J H Conway, An enumeration of knots and links, and some of their algebraic properties, from: "Computational Problems in Abstract Algebra (Proc. Conf., Oxford, 1967)", Pergamon, Oxford (1970) 329-358 MR0258014

[2] W B R Lickorish, Prime knots and tangles, Trans. Amer. Math. Soc. 267 (1981) 321332 MR621991

[3] M Scharlemann, Thin position in the theory of classical knots, from: "Handbook of knot theory", (W Menasco, M Thistlethwaite, editors), Elsevier, Amsterdam (2005) 429-459 MR2179267

[4] M Scharlemann, M Tomova, Conway products and links with multiple bridge surfaces, Michigan Math. J. 56 (2008) 113-144 MR433660 
[5] H Schubert, Über eine numerische Knoteninvariante, Math. Z. 61 (1954) 245-288 MR0072483

[6] J Schultens, Additivity of bridge numbers of knots, Math. Proc. Cambridge Philos. Soc. 135 (2003) 539-544 MR2018265

Department of Mathematics, University of California, Santa Barbara Santa Barbara, CA 93106

rcblair@math.ucsb.edu

Received: 13 May 2009 Revised: 15 December 2009 